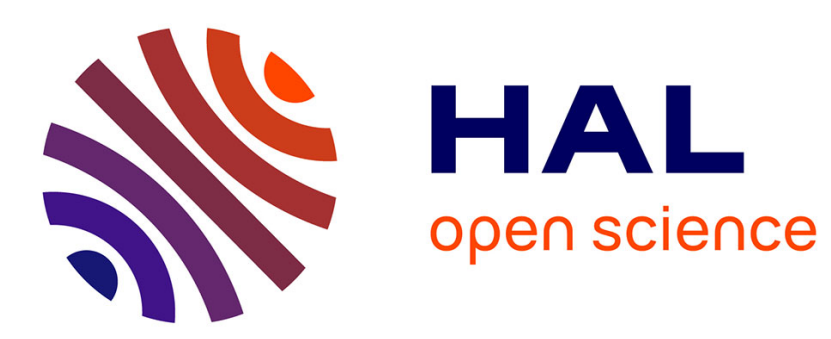

\title{
The derivation of homogenized diffusion kurtosis models for diffusion MRI
}

Houssem Haddar, Marwa Kchaou, Maher Moakher

\section{To cite this version:}

Houssem Haddar, Marwa Kchaou, Maher Moakher. The derivation of homogenized diffusion kurtosis models for diffusion MRI. Journal of Magnetic Resonance, 2018, 298, pp.48-57. hal-01945614

\section{HAL Id: hal-01945614 \\ https://hal.science/hal-01945614}

Submitted on 5 Dec 2018

HAL is a multi-disciplinary open access archive for the deposit and dissemination of scientific research documents, whether they are published or not. The documents may come from teaching and research institutions in France or abroad, or from public or private research centers.
L'archive ouverte pluridisciplinaire HAL, est destinée au dépôt et à la diffusion de documents scientifiques de niveau recherche, publiés ou non, émanant des établissements d'enseignement et de recherche français ou étrangers, des laboratoires publics ou privés. 


\title{
The Derivation of Homogenized Diffusion Kurtosis Models for Diffusion MRI
}

\author{
Houssen Haddar ${ }^{1}$, Marwa Kchaou ${ }^{2}$, and Maher Moakher ${ }^{2}$ \\ 1 INRIA, Ecole Polytechnique, Université Paris Saclay, Route de Saclay, 91128 Palaiseau Cedex, France \\ 2 Université de Tunis El Manar, Ecole Nationale d'Ingénieurs de Tunis, LAMSIN, B.P. 37, 1002 Tunis, \\ Tunisia.
}

\begin{abstract}
We use homogenization theory to establish a new macroscopic model for the complex transverse water proton magnetization in a voxel due to diffusion-encoding magnetic field gradient pulses in the case of biological tissue with impermeable membranes. In this model, new higher-order diffusion tensors emerge and offer more information about the structure of the biological tissues. We explicitly solve the macroscopic model to obtain an ordinary differential equation for the diffusion MRI signal that has similar structure as diffusional kurtosis imaging models. We finally present some validating numerical results on synthetic examples showing the accuracy of the model with respect to signals obtained by solving the Bloch-Torrey equation.
\end{abstract}

\section{Introduction}

Diffusion Magnetic Resonance Imaging (dMRI) is a non-invasive imaging technique that is applied in material sciences to investigate structural and transport properties of porous media as well as in medicine and neuroscience to study anatomical, physical, and functional properties of biological tissues and organs such as brain, muscles and bones $[7,10,16,22,27,32]$. The classical dMRI experiment consists in applying two pulsed-gradient magnetic fields with a 180 degree spin reversal between the two pulses in order to encode the displacement of water molecules between them $[5,6,21,26]$.

The aim of this paper is to propose a new justification of the use of higher order diffusion tensor in the modeling of Diffusion Tensor Imaging (DTI) (See for instance $[17,18,20]$ and the references therein), through homogenization theory [4]. Following the approach proposed in [8, 12], we consider the periodic Bloch-Torrey partial differential equation as a microscopic model for the (complex) transverse water proton magnetization diffusion due to magnetic field gradient pulses. The goal is to determine higher-order asymptotic expansion of the solution in terms of the ratio cell-size/voxel-size. The latter is assumed to be small compared to the size of a voxel in a dMRI image. The obtained model can be seen as an improvement of the one derived in $[8,17]$. We remark that the homogenization model obtained in the latter is time-independent and therefore is physically relevant only for the long-time limit of the dMRI signal. Going to higher-order terms in the homogenization process, we end up with models that depend on diffusion time and therefore would be valid for larger time intervals.

We mention here that there are other approaches to homogenization than the periodic homogenization theory used in this study. For example, in the case of random coefficients, effective medium theory can be used to obtain averaged quantities based on a statistical approach (see for instance [24] and the references therein). The latter is more general as it does not need the periodicity assumption. However, it is based on physically oriented modeling ansatz and leads to computable expressions only in specific regimes for time profile.

Higher-order homogenization expansions has been employed in many other contexts in the literature and we refer the reader to $[1,2,9,23,28]$ and the references therein for an account of the vast literature on the subject. Our main contribution is to exploit this concept to justify the so-called diffusion kurtosis imaging (DKI) proposed in the literature of dMRI (See for instance [3, $14,15,20]$. This technique is used to directly measure the non-Gaussian property of water diffusion 
characterized by a fourth-order tensor referred to as diffusion kurtosis tensor. In three-dimensional space, the kurtosis tensor has 81 components. But, owing to the full symmetry with respect to an interchange of indices, only 15 elements are independent. The DKI method provides a complementary information to DTI that can be useful in diagnosis $[15,19]$. It is based on the same type of pulse sequences employed for conventional diffusion-weighted imaging (DWI), but the required $b$ values are somewhat larger than those usually used to measure diffusion coefficients. Generalized diffusion tensors (GDTI) have been also employed in [25] in order to assess high angular resolution diffusion imaging (HARDI) data. Among the differences between GDTI and DKI is that the generalized diffusion tensors defined in [25] depend on the choice of $b$-value while the one in $[20,19]$ does not.

We shall see that our modeling lead to a DKI that depends on the time profile characteristics and therefore makes the bridge between the two mentioned above models. Moreover, we obtain computable expressions of DKI in terms of the cell geometry and intrinsic diffusion coefficients. In addition to homogenization technique, we rely on the interpretation of the macroscopic model in the Fourier domain to derive an ordinary differential equation (ODE) model that directly implies the dMRI signal over a given voxel (as for instance in $[8,11]$ ). In these first investigations, we restrict ourselves to the cases where the exchange across cell membranes is negligible, which is a good approximation for white matter or for relatively small $b$-values. The DKI expression also applies to heterogeneous intrinsic diffusion coefficients. We validate the accuracy of our models through some two-dimensional numerical simulations. We show in particular that the new model provides much better accuracy for the signal produced by simulating the full Bloch Torrey model.

Our paper is organized as follows. We present the classical Bloch-Torry model and fix notation in Section 2. We outline the periodic homogenization technique in Section 3 and provide the key steps to compute the different terms of the two-scale asymptotic expansions. We provide explicit expressions up to order 2. We present in Section 4 the ODE model that can be derived from the homogenized equations where we show that the signal from ODE model approximates the signal of the microscopic model at long diffusion times. We finally give in Section 5 some validating numerical results for toy models where we show that the signal from the homogenized model approximates the signal of the microscopic model at long diffusion times. For the reader convenience, we complement our paper with an appendix containing some technical details associated with Section 3.

\section{Bloch-Torrey equations}

For a volume $\Omega \subset \mathbb{R}^{d}$ ( $d=2$ or 3 ) occupied by a biological tissue, we denote by $\Gamma$ the union of the boundaries of all the biological cells included in $\Omega$. The domain $\Omega_{\text {ext }}$ then represents the union of two subdomains the extra-cellular, $\Omega_{e}$, and intra-cellular, $\Omega_{c}$, domains

$$
\Omega_{\mathrm{ext}}=\Omega \backslash \Gamma=\Omega_{e} \cup \Omega_{c} .
$$

The complex transverse water proton magnetization $\hat{M}=M_{x}+\iota M_{y}$, is modeled by the BlochTorrey equation $[10,29]$ :

$$
\left.\frac{\partial}{\partial t} \hat{\boldsymbol{M}}(\boldsymbol{x}, t)+\iota f(t) \boldsymbol{q} \cdot \boldsymbol{x} \hat{\boldsymbol{M}}(\boldsymbol{x}, t)-\operatorname{div}(\mathcal{D}(\boldsymbol{x}) \nabla \hat{\boldsymbol{M}}(\boldsymbol{x}, t))+\frac{1}{T_{2}} \hat{\boldsymbol{M}}(\boldsymbol{x}, t)=0, \quad \text { in } \Omega_{\mathrm{ext}} \times\right] 0, T[,
$$

where $T_{2}$ is the spin-spin relaxation time, $T>0$ is the final time, $\iota$ in the imaginary unit and $\mathcal{D}$ is the intrinsic diffusion function:

$$
\mathcal{D}= \begin{cases}\mathcal{D}_{e}, & \text { in } \Omega_{e}, \\ \mathcal{D}_{c}, & \text { in } \Omega_{c} .\end{cases}
$$

The constant vector, $\boldsymbol{q} \in \mathbb{R}^{d}$, contains the amplitude and direction of the applied diffusion-encoding magnetic field gradient multiplied by the gyro-magnetic ratio of water protons, $\gamma=2.67513 \times 10^{8}$ $\operatorname{rads}^{-1} \mathrm{~T}^{-1}$. 
If we assume that the relaxation time $T_{2}$ is a constant independent of spatial variable $\boldsymbol{x}$, then, by representing the transverse magnetisation $\hat{M}$ by a product of a purely time-dependent function $\exp \left(-\frac{1}{T_{2}} t\right)$ and a function of time and space $\boldsymbol{M}(\boldsymbol{x}, t)$, i.e.,

$$
\hat{\boldsymbol{M}}(\boldsymbol{x}, t)=\exp \left(-\frac{1}{T_{2}} t\right) \boldsymbol{M}(\boldsymbol{x}, t),
$$

the Bloch-Torrey equation (1) becomes

$$
\left.\frac{\partial}{\partial t} \boldsymbol{M}(\boldsymbol{x}, t)+\iota f(t) \boldsymbol{q} \cdot \boldsymbol{x} \boldsymbol{M}(\boldsymbol{x}, t)-\operatorname{div}(\mathcal{D}(\boldsymbol{x}) \nabla \boldsymbol{M}(\boldsymbol{x}, t))=0, \quad \text { in } \Omega_{\mathrm{ext}} \times\right] 0, T[.
$$

Although that, in a very recent study, Veraart et al. [30] showed that the relaxation time $T_{2}$ depends on the spatial position and hence it might not be valid to assume that $T_{2}$ is spatially uniform, in this article we make this assymption.

The time profile, $f(t)$ is chosen to satisfy the refocusing (or rephasing) condition

$$
\int_{0}^{T_{E}} f(t) \mathrm{d} t=0
$$

where $T_{E}$ is the time at which the signal is measured and is called the echo time. In this work, we consider the case where there is no water exchange between compartments, i.e., we consider the boundary condition

$$
\left.\mathcal{D}(\boldsymbol{x}) \nabla \boldsymbol{M}(\boldsymbol{x}, t) \cdot \boldsymbol{\nu}\right|_{\Gamma}=0, \quad \text { on } \Gamma,
$$

where $\boldsymbol{\nu}$ is the outward unit normal to the domain $\Omega_{c}$. As a matter of fact, the diffusion inside and outside the cells are decoupled and one can only consider the diffusion in one domain (the other one is obtained in the same way). The PDE (3) and the boundary condition (4) must be supplemented with the initial condition:

$$
\boldsymbol{M}(\boldsymbol{x}, 0)=\boldsymbol{M}_{\text {init }}, \quad \text { in } \Omega_{\text {ext }} .
$$

The dMRI signal at echo time $T_{E}$ is the integral of magnetization $\boldsymbol{M}\left(\boldsymbol{x}, T_{E}\right)$, normalized by the mass:

$$
S:=\frac{1}{|\Omega|} \int_{\Omega} \boldsymbol{M}\left(\boldsymbol{x}, T_{E}\right) \mathrm{d} \boldsymbol{x},
$$

where $\boldsymbol{M}$ is the solution of equation (3) and $|\Omega|$ denotes the volume of $\Omega$. Numerically, the signal is usually plotted against a quantity called the $b$-value

$$
b(|\boldsymbol{q}|):=|\boldsymbol{q}|^{2} \int_{0}^{T_{E}}\left(\int_{0}^{t} f(s) \mathrm{d} s\right)^{2} \mathrm{~d} t .
$$

The homogenization method is a mathematical theory of averaging. It is an approach that allows to infer variations at macroscopic scales (pixel-level) from microscopic scale (cellular-level) description of the PDE model. For simplicity, we shall employ periodic homogenization theory $[2$, 4]. We assume that the volume to be modeled, $\Omega$, can be described at the microscopic scale as a periodic domain. The periodicity scale $\varepsilon$, related to the size of cell versus the size of the voxel, is assumed to be small. We define the normalized periodicity box $Y=] 0,1\left[^{d}\right.$ such that $Y=Y_{e} \cup Y_{c}$ and $\Gamma_{m}=\partial Y_{c}=\partial Y_{e} \backslash \partial \bar{Y}$. As indicated earlier, due to the choice of the boundary conditions at the interface, applying the homogenization process to the exterior or the interior domain is similar. However, if the intra-cellular domain does not communicate with the extra-cellular domain and has a geometry $Y_{e}$ strictly included inside $Y$, then the homogenized tensor associated with this compartment vanishes. Therefore, in what follow we concentrate on applying the homogenization process to one subdomain that is the exterior domain. Taking into account the periodicity at microscale we define

$$
\Xi_{\varepsilon}=\left\{\xi \in \mathbb{Z}^{d} \mid \varepsilon(\xi+Y) \cap \Omega \neq \emptyset\right\}, \Omega_{e}^{\varepsilon}=\bigcup_{\xi \in \Xi}\left\{\varepsilon\left(\xi+Y_{e}\right) \cap \Omega\right\} .
$$


Therefore, the initial boundary-value problem (3), (4) and (5) can be rewritten as:

$$
\begin{cases}\frac{\partial}{\partial t} \boldsymbol{M}_{\varepsilon}(\boldsymbol{x}, t)+\iota f(t) Q(\boldsymbol{x}) \boldsymbol{M}_{\varepsilon}(\boldsymbol{x}, t)-\operatorname{div}\left(\mathcal{D}_{\varepsilon}(\boldsymbol{x}) \nabla \boldsymbol{M}_{\varepsilon}(\boldsymbol{x}, t)\right)=0, & \text { in } \left.\Omega_{e}^{\varepsilon} \times\right] 0, T[, \\ \mathcal{D}_{\varepsilon}(\boldsymbol{x}) \nabla \boldsymbol{M}_{\varepsilon}(\boldsymbol{x}, t) \cdot \boldsymbol{\nu}=0, & \text { on } \Xi_{\varepsilon}, \\ \boldsymbol{M}_{\varepsilon}(\cdot, 0)=\boldsymbol{M}_{\text {init }}, & \text { in } \Omega_{e}^{\varepsilon},\end{cases}
$$

where $\mathcal{D}_{\varepsilon}(\boldsymbol{x}):=\mathcal{D}\left(\frac{\boldsymbol{x}}{\varepsilon}\right)$ and $Q(\boldsymbol{x})=\boldsymbol{q} \cdot \boldsymbol{x}$. We assume that the initial data $\boldsymbol{M}_{\text {init }}$ defined on $\Omega$ is independent of $\varepsilon$.

For the choice of the volume to be modeled we shall take $\Omega=\mathbb{R}^{d}$ to avoid complications associated with imposing boundary conditions on $\Omega$. This is justified by the fact that the voxel of interest is itself surrounded by other voxels and diffusion time is such that diffusion length is smaller than the voxel dimensions. This assumption avoids the modeling of boundary layer effects and justifies the use of higher-order asymptotics in the periodic homogenization ansatz.

\section{A macroscopic model through two-scale asymptotic expansion}

The present section is dedicated to an outline of the periodic homogenization machinery for deriving macroscopic models. We are interested in models that involve higher-order diffusion tensors, which requires going at least up to the second order in the asymptotic expansion. This type of calculation is by now very classical in the mathematical literature and its justification is well understood in the setting where no macroscopic boundary conditions are involved. The specificity of the considered model with respect to the literature is the presence of the term $\iota f(t) Q(\boldsymbol{x}) \boldsymbol{M}_{\varepsilon}$ which induces new homogenized tensors for the second-order model as compared to the classical ones (see for instance [8]).

Following the periodic homogenization $[4,28]$, we postulate a two-scale asymptotic expansion for $\boldsymbol{M}_{\varepsilon}$ in $\Omega_{e}^{\varepsilon}$ as

$$
\boldsymbol{M}_{\varepsilon}(\boldsymbol{x}, t)=\sum_{n=0}^{+\infty} \varepsilon^{n} \boldsymbol{M}_{n}\left(\boldsymbol{x}, \frac{\boldsymbol{x}}{\varepsilon}, t\right) \quad \text { in } \Omega_{e}^{\varepsilon},
$$

where the function $\boldsymbol{M}_{n}(\boldsymbol{x}, \boldsymbol{y}, t)$ is defined on $\left.\Omega \times Y_{e} \times\right] 0, T$ [ and is $Y$-periodic in $\boldsymbol{y}$ (the variable $\boldsymbol{x}$ indicates macroscopic variations and the variable $\boldsymbol{y}=\frac{\boldsymbol{x}}{\varepsilon}$ indicates the microscopic ones). Our goal is to characterize term by term the functions $\boldsymbol{M}_{n}$ up to $n=2$ and then write a model for the truncated sum (up to $n=2$ ) of the average with respect to the $\boldsymbol{y}$ variable. This would give us (a formally) second-order approximation of $\boldsymbol{M}_{\varepsilon}$ with respect to $\varepsilon$. For second-order PDE, in order to obtain the macroscopic equations for $n=2$ we need to write the microscopic equations that involve the terms up to the order $n+2$.

In the following we shall use the notation

$$
\widehat{\boldsymbol{M}}_{n}(\boldsymbol{x}, t)=\left\langle\boldsymbol{M}_{n}(\boldsymbol{x}, \cdot, t)\right\rangle_{Y_{e}}=\frac{1}{\left|Y_{e}\right|} \int_{Y_{e}} \boldsymbol{M}_{n}(\boldsymbol{x}, \boldsymbol{y}, t) d \boldsymbol{y}
$$

Also, in order to shorten the formulas, we respectively denote by $: \therefore$ and $::$ the contraction products with respect to the last 2, 3 and 4 indices of the given two tensors. For instance, for any fourth-order tensors $\mathcal{C}$ and $\mathcal{P}$

$$
(\mathcal{C}: \mathcal{P})_{i, l}=\sum_{j, k} \mathcal{C}_{i l j k} \mathcal{P}_{i l j k}, \quad(\mathcal{C} \therefore \mathcal{P})_{i}=\sum_{l, j, k} \mathcal{C}_{i l j k} \mathcal{P}_{i l j k} \text { and }(\mathcal{C}:: \mathcal{P})=\sum_{i, l, j, k} \mathcal{C}_{i l j k} \mathcal{P}_{i l j k}
$$

The tensor product of two vectors $\boldsymbol{u}$ and $\boldsymbol{v}$ is defined by

$$
(\boldsymbol{u} \otimes \boldsymbol{v})_{i j}=u_{i} v_{j}
$$




\subsection{Macroscopic model for $\widehat{M}_{0}, \widehat{M}_{1}$ and $\widehat{M}_{2}$}

We here summarize the equations satisfied by $\widehat{\boldsymbol{M}}_{0}, \widehat{\boldsymbol{M}}_{1}$ and $\widehat{\boldsymbol{M}}_{2}$ which are obtained after substituting (7) into (6) and equating the same powers of $\varepsilon$. The formal procedure is detailed in Sections A.1-A.3 of the Appendix. These equations necessitate the calculation of some macroscopic quantities through the solutions of time-independent problems posed on the unit box $Y$. We first introduce the solutions to the following classical unit-box problems

$$
\begin{cases}-\operatorname{div}_{\boldsymbol{y}}\left(\mathcal{D} \nabla_{\boldsymbol{y}} \omega_{i}+\sigma \boldsymbol{e}_{i}\right)=0, & \text { in } Y_{e} \\ \mathcal{D} \nabla_{\boldsymbol{y}} \omega_{i} \cdot \boldsymbol{\nu}+\mathcal{D} \boldsymbol{e}_{i} \cdot \boldsymbol{\nu}=0, & \text { on } \Gamma_{m} \\ \omega_{i} \text { is } Y \text {-periodic, }, \quad\left\langle\omega_{i}\right\rangle_{Y_{e}}=0 & \end{cases}
$$

where $\boldsymbol{e}_{i}, i=1, \ldots, d$ are the vectors of the canonical basis of $\mathbb{R}^{d}$. These solutions allow us to define the homogenized second-order tensor $\mathbb{D}$ by

$$
\mathbb{D}_{i j}:=\left\langle\mathcal{D} \nabla \omega_{j} \cdot \boldsymbol{e}_{i}+\mathcal{D} \delta_{i j}\right\rangle_{Y_{e}}, \quad i, j=1, \ldots, d .
$$

We observe that $\mathbb{D}$ is symmetric as its components can be written as $\mathbb{D}_{i j}=\left\langle\mathcal{D}\left(\nabla \omega_{j}+\boldsymbol{e}_{j}\right) \cdot\left(\nabla \omega_{i}+\right.\right.$ $\left.\left.\boldsymbol{e}_{i}\right)\right\rangle_{Y_{e}}$. With the aid of this tensor we can write the homogenized equations for $\widehat{\boldsymbol{M}}_{0}$ as

$$
\begin{cases}\frac{\partial}{\partial t} \widehat{\boldsymbol{M}}_{0}+\iota Q f(t) \widehat{\boldsymbol{M}}_{0}-\operatorname{div}_{\boldsymbol{x}}\left(\mathbb{D} \nabla_{\boldsymbol{x}} \widehat{\boldsymbol{M}}_{0}\right)=0, & \text { in } \Omega \times] 0, T[ \\ \overline{\boldsymbol{M}}_{0}(\cdot, 0)=\boldsymbol{M}_{\text {init }}, & \text { in } \Omega .\end{cases}
$$

It turns out that, likewise, the macroscopic model for $\widehat{\boldsymbol{M}}_{1}$ can also be written as

$$
\begin{cases}\frac{\partial}{\partial t} \widehat{\boldsymbol{M}}_{1}+\iota Q f(t) \widehat{\boldsymbol{M}}_{1}-\operatorname{div}_{\boldsymbol{x}}\left(\mathbb{D} \nabla_{\boldsymbol{x}} \widehat{\boldsymbol{M}}_{1}\right)=0, & \text { in } \Omega \times] 0, T[ \\ \widehat{\boldsymbol{M}}_{1}(\cdot, 0)=\mathbf{0}, & \text { in } \Omega .\end{cases}
$$

The macroscopic model for $\widehat{\boldsymbol{M}}_{2}$ involve two additional homogenized tensors and can be written as

$$
\begin{cases}\frac{\partial}{\partial t} \widehat{\boldsymbol{M}}_{2}+\iota Q f(t) \widehat{\boldsymbol{M}}_{2}-\operatorname{div}_{\boldsymbol{x}}\left(\mathbb{D} \nabla_{\boldsymbol{x}} \widehat{\boldsymbol{M}}_{2}\right)=\operatorname{div}_{\boldsymbol{x}}\left(\mathbb{T} \therefore \nabla_{\boldsymbol{x}}^{3} \widehat{\boldsymbol{M}}_{0}\right) & \\ \quad-\operatorname{div}_{\boldsymbol{x}}\left(\mathbb{D} \nabla_{\boldsymbol{x}}\left(\mathbb{S}: \nabla_{\boldsymbol{x}}^{2} \widehat{\boldsymbol{M}}_{0}\right)\right)+\iota f(t) \mathbb{S}: \boldsymbol{q} \otimes \nabla_{\boldsymbol{x}} \widehat{\boldsymbol{M}}_{0}, & \text { in } \Omega \times] 0, T[, \\ \widehat{\boldsymbol{M}}_{2}(\cdot, 0)=\mathbf{0}, & \text { in } \Omega,\end{cases}
$$

where the fourth-order tensor $\mathbb{T}$ is defined by

$$
\mathbb{T}_{i j k l}=\left\langle\mathcal{D} \frac{\partial}{\partial y_{l}}\left(\mathcal{Q}_{i j k}+\mathcal{R}_{i j k}\right)\right\rangle_{Y_{e}}, \quad i, j, k, l=1, \ldots, d,
$$

and the symmetric second-order tensor $\mathbb{S}$ is defined by

$$
\mathbb{S}_{i j}=\left\langle\mathcal{D} \frac{\partial}{\partial y_{j}} \theta_{i}\right\rangle_{Y_{e}}=-\left\langle\omega_{i} \cdot \omega_{j}\right\rangle_{Y_{e}} .
$$

The functions $\theta_{i}, \mathcal{Q}_{i j k}$ and $\mathcal{R}_{i j k}$ for $i, j, k=1, \ldots, d$ are respectively defined as solutions to the following problems

$$
\begin{aligned}
& \begin{cases}-\operatorname{div}_{\boldsymbol{y}}\left(\mathcal{D} \nabla_{\boldsymbol{y}} \theta_{i}\right)=\omega_{i}, & \text { in } Y_{e}, \\
\mathcal{D} \nabla_{\boldsymbol{y}} \theta_{i} \cdot \nu=0, & \text { on } \Gamma_{m}, \\
\theta_{i} \text { is } Y \text {-periodic, }, \quad\left\langle\theta_{i}\right\rangle_{Y_{e}}=0 . & \end{cases} \\
& \begin{cases}-\operatorname{div}_{\boldsymbol{y}}\left(\mathcal{D} \nabla_{\boldsymbol{y}} \mathcal{Q}_{i j k}\right)=\mathcal{D} \omega_{j} \delta_{k i}+\mathcal{D} \frac{\partial}{\partial y_{k}}\left(\chi_{i j}+\varphi_{i j}\right)-\mathbb{H}_{i j k}, \text { in } Y_{e}, & \text { in } Y_{e}, \\
\mathcal{D} \nabla_{\boldsymbol{y}} \mathcal{Q}_{i j k} \cdot \boldsymbol{\nu}=0, & \text { on } \Gamma_{m} \\
\mathcal{Q}_{i j k} \text { is } Y \text {-periodic, }, \quad\left\langle\mathcal{Q}_{i j k}\right\rangle_{Y_{e}}=0 & \end{cases} \\
& \begin{cases}-\operatorname{div}_{\boldsymbol{y}}\left(\mathcal{D} \nabla_{\boldsymbol{y}} \mathcal{R}_{i j k}\right)=\frac{\partial}{\partial y_{k}}\left(\mathcal{D}\left(\chi_{i j}+\varphi_{i j}\right)\right), & \text { in } Y_{e}, \\
\mathcal{D} \nabla_{\boldsymbol{y}} \mathcal{R}_{i j k} \cdot \boldsymbol{\nu}+\mathcal{D}\left(\chi_{i j}+\mathcal{D} \varphi_{i j}\right) \nu_{k}=0, & \text { on } \Gamma_{m}, \\
\mathcal{R}_{i j k} \text { is } Y \text {-periodic, },\left\langle\mathcal{R}_{i j k}\right\rangle_{Y_{e}}=0 . & \end{cases}
\end{aligned}
$$


These problems make use of the functions $\chi_{i j}$ and $\varphi_{i j} i, j=1, \ldots, d$ that are defined as solutions to the problems

$$
\begin{aligned}
& \begin{cases}-\operatorname{div}_{\boldsymbol{y}}\left(\mathcal{D} \nabla_{\boldsymbol{y}} \chi_{i j}\right)=\frac{\partial}{\partial y_{j}}\left(\mathcal{D} \omega_{i}\right), & \text { in } Y_{e}, \\
\mathcal{D} \nabla_{\boldsymbol{y}} \chi_{i j} \cdot \boldsymbol{\nu}+\mathcal{D} \omega_{i} \nu_{j}=0, & \text { on } \Gamma_{m}, \\
\chi_{i j} \text { is } Y \text {-periodic, },\left\langle\chi_{i j}\right\rangle_{Y_{e}}=0, & \end{cases} \\
& \begin{cases}-\operatorname{div}_{\boldsymbol{y}}\left(\mathcal{D} \nabla_{\boldsymbol{y}} \varphi_{i j}\right)=\mathcal{D} \frac{\partial}{\partial y_{i}} \omega_{j}+\mathcal{D} \delta_{i j}-\mathbb{D}_{i j}, & \text { in } Y_{e}, \\
\mathcal{D} \nabla_{\boldsymbol{y}} \varphi_{i j} \cdot \boldsymbol{\nu}=0, & \text { on } \Gamma_{m}, \\
\varphi_{i j} \text { is } Y \text {-periodic, }, \quad\left\langle\varphi_{i j}\right\rangle_{Y_{e}}=0 . & \end{cases}
\end{aligned}
$$

We refer to Section A.4 in the appendix for the expression of the third order tensor $\mathbb{H}$.

\subsection{The second-order macroscopic model}

Using the three macroscopic models for $\widehat{\boldsymbol{M}}_{0}, \widehat{\boldsymbol{M}}_{1}$ and $\widehat{\boldsymbol{M}}_{2}$ given in (9), (10) and (11), we obtain the following second-order model that is satisfied, up to $O\left(\varepsilon^{3}\right)$

$$
\begin{cases}\frac{\partial}{\partial t} \boldsymbol{M}_{\varepsilon, 2}-\operatorname{div}_{\boldsymbol{x}}\left(\mathbb{D} \nabla_{\boldsymbol{x}}\left(1-\varepsilon^{2} \mathbb{S}: \nabla_{\boldsymbol{x}}^{2}\right) \boldsymbol{M}_{\varepsilon, 2}\right) & \\ -\varepsilon^{2} \operatorname{div}_{\boldsymbol{x}}\left(\mathbb{T} \therefore \nabla_{\boldsymbol{x}}^{3} \boldsymbol{M}_{\varepsilon, 2}\right)+\iota f(t)\left(Q \boldsymbol{M}_{\varepsilon, 2}-\varepsilon^{2} \mathbb{S}: \boldsymbol{q} \otimes \nabla_{\boldsymbol{x}} \boldsymbol{M}_{\varepsilon, 2}\right)=0, & \text { in } \Omega \times] 0, T[, \\ \boldsymbol{M}_{\varepsilon, 2}(\cdot, 0)=\boldsymbol{M}_{\text {init }}, & \text { in } \Omega .\end{cases}
$$

We then formally expect that

$$
\boldsymbol{M}_{\varepsilon}=M_{\varepsilon, 2}+O\left(\varepsilon^{3}\right) \quad \text { in } \Omega_{e}^{\varepsilon} .
$$

The model (17) is in fact unstable for computing $\boldsymbol{M}_{\varepsilon, 2}$ since the fourth-order operator is non negative [1,9]. However, since we are only interested in the mean value of $\boldsymbol{M}_{\varepsilon, 2}$ at echo time, one is able to deduce from this model an ODE for the mean value that is stable for sufficiently small $\varepsilon$ (see next section).

Remark 1. We observe that the homogenized model (17) is independent from the choice of the measuring unit (m, mm, $\mu \mathrm{m}$, etc.) of the unit box $Y$. For instance, if one replaces $Y$ by $Y^{\prime}=\eta Y$ where $\eta$ is a scaling factor, then one should change $\epsilon$ by $\varepsilon / \eta$ (since the physical periodicity is $\varepsilon Y$ ). If we denote by $\mathbb{D}_{\eta}, \mathbb{S}_{\eta}$ and $\mathbb{T}_{\eta}$ the homogenized tensors computed by replacing $Y$ with $Y^{\prime}$, then one can easily check that

$$
\mathbb{D}_{\eta}=\mathbb{D}, \quad \mathbb{S}_{\eta}=\eta^{2} \mathbb{S}, \quad \text { and } \quad \mathbb{T}_{\eta}=\eta^{2} \mathbb{T} .
$$

This shows that the coefficients in (17) remain the same under the scaling $\eta$.

\section{Asymptotic dMRI signal model for DKI}

In this section, we explain how to use the macroscopic model (17) to obtain an ODE model that allows us to compute the Kurtosis tensor. This approach has also been used in [8] for DTI and in [11] to obtain kurtosis approximations for small diffusion times. In practice, the reference normalized signal is measured at the echo time $T_{E}$ and it is given by

$$
S_{\varepsilon}^{B T}(\boldsymbol{q}):=\frac{\int_{\mathbb{R}^{d}} \boldsymbol{M}_{\varepsilon}\left(\boldsymbol{x}, T_{E}\right) \mathrm{d} \boldsymbol{x}}{\int_{\mathbb{R}^{d}} \boldsymbol{M}_{\mathrm{init}} \mathrm{d} \boldsymbol{x}},
$$

where $\boldsymbol{M}_{\varepsilon}$ is the solution of the reference Bloch-Torrey equation (6). Then, it is natural to define the approximate normalized signal by

$$
\widetilde{S}_{\varepsilon}(\boldsymbol{q}):=\frac{\int_{\mathbb{R}^{d}} \boldsymbol{M}_{\varepsilon, 2}\left(\boldsymbol{x}, T_{E}\right) \mathrm{d} \boldsymbol{x}}{\int_{\mathbb{R}^{d}} \boldsymbol{M}_{\mathrm{init}} \mathrm{d} \boldsymbol{x}} .
$$


We start by transforming the third-order equation (17) by introducing the new unknown variable $\widetilde{\boldsymbol{M}}_{\varepsilon, 2}$ defined almost everywhere on $\left.\mathbb{R}^{d} \times\right] 0, T[$ by

$$
\widetilde{\boldsymbol{M}}_{\varepsilon, 2}(\boldsymbol{x}, t)=\boldsymbol{M}_{\varepsilon, 2}(\boldsymbol{x}, t) e^{\iota q \boldsymbol{x} \cdot \boldsymbol{n} F(t)},
$$

where $F(t):=\int_{0}^{t} f(s) d s$ and $\boldsymbol{n}$ is a unit vector such that $\boldsymbol{q}=q \boldsymbol{n}$. Since $F\left(T_{E}\right)=0$, one observes that $\widetilde{\boldsymbol{M}}_{\varepsilon, 2}\left(\cdot, T_{E}\right)=\boldsymbol{M}_{\varepsilon, 2}\left(\cdot, T_{E}\right)$ and therefore

$$
\widetilde{S}_{\varepsilon}(\boldsymbol{q}):=\frac{\int_{\mathbb{R}^{d}} \widetilde{\boldsymbol{M}}_{\varepsilon, 2}\left(\boldsymbol{x}, T_{E}\right) \mathrm{d} \boldsymbol{x}}{\int_{\mathbb{R}^{d}} \boldsymbol{M}_{\mathrm{init}} \mathrm{d} \boldsymbol{x}} .
$$

Setting

$$
m_{\varepsilon}(t):=\int_{\mathbb{R}^{d}} \widetilde{\boldsymbol{M}}_{\varepsilon, 2}\left(\boldsymbol{x}, T_{E}\right) \mathrm{d} \boldsymbol{x},
$$

one obtains after inserting the change of variable (18) in (17) and integrating over $\mathbb{R}^{d}$

$$
\left\{\begin{aligned}
\frac{d}{d t} m_{\varepsilon}(t)+F(t)^{2} q^{2}(\mathbb{D}:(\boldsymbol{n} \otimes \boldsymbol{n})) m_{\varepsilon}(t) & +\varepsilon^{2} F(t)^{4} q^{4}(\mathbb{B}::(\boldsymbol{n} \otimes \boldsymbol{n} \otimes \boldsymbol{n} \otimes \boldsymbol{n})) m_{\varepsilon}(t) \\
& -\varepsilon^{2} f(t) F(t) q^{2}(\mathbb{S}:(\boldsymbol{n} \otimes \boldsymbol{n})) m_{\varepsilon}(t)=0,
\end{aligned}\right.
$$

where the fourth-order tensor $\mathbb{B}$, called the Burnett tensor [9], is defined by

$$
\mathbb{B}_{i j k l}=\mathbb{D}_{i j} \mathbb{S}_{k l}-\mathbb{T}_{i j k l} .
$$

We see clearly that the ODE model (19) can be solved explicitly as

$$
m_{\varepsilon}(t)=m_{\varepsilon}(0) \exp \left(-\int_{0}^{t} L_{\varepsilon}(\boldsymbol{q}, s) d s\right)
$$

where

$$
\begin{aligned}
L_{\varepsilon}(\boldsymbol{q}, t)= & F(t)^{2} q^{2}(\mathbb{D}:(\boldsymbol{n} \otimes \boldsymbol{n}))+\varepsilon^{2} F(t)^{4} q^{4}(\mathbb{B}::(\boldsymbol{n} \otimes \boldsymbol{n} \otimes \boldsymbol{n} \otimes \boldsymbol{n})) \\
& -\varepsilon^{2} f(t) F(t) q^{2}(\mathbb{S}:(\boldsymbol{n} \otimes \boldsymbol{n})) .
\end{aligned}
$$

The approximate normalized signal can then be rewritten as

$$
\widetilde{S}_{\varepsilon}(\boldsymbol{q})=\frac{m_{\varepsilon}\left(T_{E}\right)}{m_{\varepsilon}(0)}=\exp \left(-\int_{0}^{T_{E}} L_{\varepsilon}(\boldsymbol{q}, s) d s\right) .
$$

We observe that $\int_{0}^{T_{E}} f(t) F(t) d t=\left(F\left(T_{E}\right)^{2}-F(0)^{2}\right) / 2=0$ and therefore the expression simplifies to

$$
\widetilde{S}_{\varepsilon}(\boldsymbol{q})=\exp \left(-K_{\varepsilon}\left(\boldsymbol{q}, T_{E}\right)\right),
$$

where

$$
K_{\varepsilon}\left(\boldsymbol{q}, T_{E}\right):=b(q)(\mathbb{D}:(\boldsymbol{n} \otimes \boldsymbol{n}))+\varepsilon^{2} q^{4}(\mathbb{B}::(\boldsymbol{n} \otimes \boldsymbol{n} \otimes \boldsymbol{n} \otimes \boldsymbol{n})) \int_{0}^{T_{E}} F(t)^{4} d t .
$$

The expression for $-\ln \left(\widetilde{S}_{\varepsilon}(\boldsymbol{q})\right)$ resembles the one proposed in [15] for DKI. The fourth-order tensor $\mathbb{W}$ referred to as diffusion kurtosis tensor is defined in [15] such that

$$
\ln \left[\widetilde{S}_{\varepsilon}(b, \boldsymbol{n})\right]=-b(q)(\mathbb{D}:(\boldsymbol{n} \otimes \boldsymbol{n}))+\frac{1}{6} b(q)^{2}\left(\frac{1}{3} \operatorname{Tr} \mathbb{D}\right)^{2}(\mathbb{W}::(\boldsymbol{n} \otimes \boldsymbol{n} \otimes \boldsymbol{n} \otimes \boldsymbol{n})) .
$$

Then, according to our model, the fourth-order diffusion kurtosis tensor $\mathbb{W}$ and the fourth-order Burnett tensor $\mathbb{B}$ are multiple of one another and are related by

$$
\mathbb{W}=-6 \varepsilon^{2} \frac{\int_{0}^{T_{E}} F(t)^{4} d t}{\left(\frac{1}{3} \operatorname{Tr} \mathbb{D}\right)^{2}\left(\int_{0}^{T_{E}} F(t)^{2} d t\right)^{2}} \mathbb{B} .
$$


In addition to having explicit dependence on the microstructure geometry, we observe that this expression depends also on the time profile $f$ through $\Delta$ and $\delta$. The latter is a notable difference with $[15,19,31]$.

We observe that for fixed $\boldsymbol{q}, K_{\varepsilon}\left(\boldsymbol{q}, T_{E}\right)$ is indeed an approximation of $b(q) \mathbb{D} \boldsymbol{n} \cdot \boldsymbol{n}$ as $\varepsilon \rightarrow 0$. However, this approximation is not uniform with respect to $q \rightarrow \infty$. For instance, one can prove that

$$
(\mathbb{B}::(\boldsymbol{n} \otimes \boldsymbol{n} \otimes \boldsymbol{n} \otimes \boldsymbol{n})) \leq 0,
$$

exactly in the same way as it is proved in [9] for the case $\Gamma_{m}=\emptyset$. Therefore, $K_{\varepsilon}\left(\boldsymbol{q}, T_{E}\right)$ can become negative for large $q$ and fixed $\varepsilon$. A domain of validity can be roughly fixed by the values of $q$ and $\varepsilon$ such that $K_{\varepsilon}\left(\boldsymbol{q}, T_{E}\right)>0$. One can also replace $K_{\varepsilon}\left(\boldsymbol{q}, T_{E}\right)$ by the Padé approximation for small $\varepsilon$, namely

$$
\tilde{K}_{\varepsilon}\left(\boldsymbol{q}, T_{E}\right)=\frac{(b(q)(\mathbb{D}:(\boldsymbol{n} \otimes \boldsymbol{n})))^{2}}{b(q)(\mathbb{D}:(\boldsymbol{n} \otimes \boldsymbol{n}))-\varepsilon^{2} q^{4} \int_{0}^{T_{E}} F(t)^{4} d t(\mathbb{B}::(\boldsymbol{n} \otimes \boldsymbol{n} \otimes \boldsymbol{n} \otimes \boldsymbol{n}))} .
$$

We observe that $K_{\varepsilon}\left(\boldsymbol{q}, T_{E}\right)=\tilde{K}_{\varepsilon}\left(\boldsymbol{q}, T_{E}\right)+O\left(\varepsilon^{4}\right)$. Therefore, while this substitution does not affect the accuracy of the model in terms of $\varepsilon$ it leads to a stable signal for all values of $\varepsilon$ and $q$.

\section{$5 \quad$ Numerical results}

Our objective in this numerical section is to measure the accuracy of the second-order model and the improvement it yields over using the zeroth-order model corresponding to $\varepsilon=0$. Our reference solution will be provided by numerical solution of the full Bloch-Torrey model (6). Since our concern is to measure the accuracy of the model, using $K_{\varepsilon}$ or $\tilde{K}_{\varepsilon}$ is the same as long as we restrict ourselves with $K_{\varepsilon}>0$. We shall use the classical Pulsed Gradient Spin Echo (PGSE) sequence, with two rectangular pulses of duration $\delta$, separated by a time interval $\Delta-\delta$, see e.g. [21]:

$$
f(t)= \begin{cases}1, & \text { if } 0<t \leq \delta \\ -1, & \text { if } \Delta<t \leq \Delta+\delta \\ 0, & \text { elsewhere }\end{cases}
$$

We assume that the RF pulse is produced at $t=\frac{\delta+\Delta}{2}$. Consequently, we take the echo time $T_{E}=\delta+\Delta$.

For this time profile

$$
b(q)=q^{2} \int_{0}^{T_{E}} F(t)^{2} d t=q^{2} \delta^{2}\left(\Delta-\frac{\delta}{3}\right)
$$

and

$$
\int_{0}^{T_{E}} F(t)^{4} d t=\delta^{4}\left(\Delta-\frac{3}{5} \delta\right)
$$

We then deduce that

$$
K_{\varepsilon}\left(\boldsymbol{q}, T_{E}\right)=b(q)(\mathbb{D}:(\boldsymbol{n} \otimes \boldsymbol{n}))+\varepsilon^{2} b(q)^{2}(\mathbb{B}::(\boldsymbol{n} \otimes \boldsymbol{n} \otimes \boldsymbol{n} \otimes \boldsymbol{n})) \frac{\left(\Delta-\frac{3}{5} \delta\right)}{\left(\Delta-\frac{\delta}{3}\right)^{2}} .
$$

and the coefficients of the diffusion kurtosis tensor are given by

$$
\mathbb{W}_{i j k l}=-6 \varepsilon^{2} \frac{\left(\Delta-\frac{3}{5} \delta\right)}{\left(\Delta-\frac{\delta}{3}\right)^{2}\left(\frac{1}{3} \operatorname{Tr} \mathbb{D}\right)^{2}} \mathbb{B}_{i j k l} .
$$

Therefore, to obtain the approximate normalized signal, $\widetilde{S}_{\varepsilon}$, we need to compute the tensors $\mathbb{D}$ and $\mathbb{B}$ by solving the box-problems (8), (13) and (14). For the numerical tests presented in the following section, the solutions to these problem is numerically evaluated using the finite element solver FreeFem++ [13]. 
In order to solve the Bloch-Torrey model (6), we use the same change of unknown as (18), namely

$$
\widetilde{\boldsymbol{M}}_{\varepsilon}(\boldsymbol{x}, t)=\boldsymbol{M}_{\varepsilon}(\boldsymbol{x}, t) e^{\iota q \boldsymbol{x} \cdot \boldsymbol{n} F(t)} .
$$

We impose constant initial magnetization $\boldsymbol{M}_{\text {init }}$. Since the coefficients in the equation satisfied by $\widetilde{\boldsymbol{M}}_{\varepsilon}$ are all periodic, the solution is also periodic and therefore the computational domain can be restricted to one period $Y_{\varepsilon}=\varepsilon Y$ (with periodicity conditions on the boundary). The normalized signal is then computed as

$$
S_{\varepsilon}^{B T}(\boldsymbol{q})=\frac{\int_{Y_{\varepsilon}} \widetilde{\boldsymbol{M}}_{\varepsilon}\left(\boldsymbol{x}, T_{E}\right) \mathrm{d} \boldsymbol{x}}{\left|Y_{\varepsilon}\right| \boldsymbol{M}_{\mathrm{init}}}
$$

For the numerical simulations, once again we use the finite-element solver FreeFem $++[13]$ with a Crank-Nicolson scheme for time stepping to evaluate the solution to the full Bloch-Torrey model and the associated normalized signal $S_{\varepsilon}^{B T}(\boldsymbol{q})$. All our numerical examples are restricted to $2 \mathrm{D}$ configurations $(d=2)$.

\subsection{Examples with impermeable cells}

We consider two examples of geometries where we vary the number of impermeable components inside a period. Figure (1) shows the configurations that we choose and the finite-element mesh that has been used to compute the solution for the Bloch-Torrey model (6) as well as the solutions for the different problems associated with the homogenized tensors.

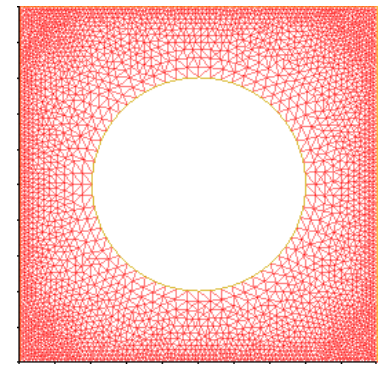

(a)

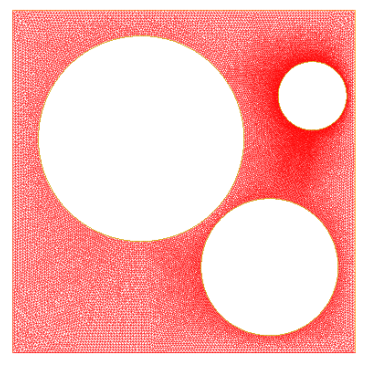

(b)

Fig. 1. Periodicity box $Y$ of $1 \mathrm{~mm}^{2}$ and associated finite-element mesh for the two different configurations of impermeable cells.

Figure 2 displays, for each geometry in Figure 1, $-\ln (S)$ according to $\varepsilon$ for three signals the reference signal, $S_{\varepsilon}^{B T}$, the approximate signal for zero order, $\widetilde{S_{0}}$ and the approximate signal for order two, $\widetilde{S_{\varepsilon}}$, where the values of the model parameters are indicated. The curve $-\ln \left(\widetilde{S_{0}}\right)$ is the horizontal line at $b(q) \mathbb{D}_{2,2}$ that corresponds to the limiting problem as $\varepsilon$ goes to 0 . One clearly observes the improvement of the second-order model over this zeroth-order approximation for the three configurations. This indeed validates the expressions derived for the second-order model and attest the relevance of using DKI for improving dMRI imaging.

Figure 3 displays the signal in terms of the $b$-value for fixed value of $\varepsilon$ and for the geometry configuration indicated in Figure 1(b). We show the results obtained for different values of the parameter $\Delta=2.5,5 \mathrm{~ms}$. These examples show that our approximate kurtosis model has the correct dependence on the parameter $\Delta$ since we obtain a better approximation of the signal for each case. 


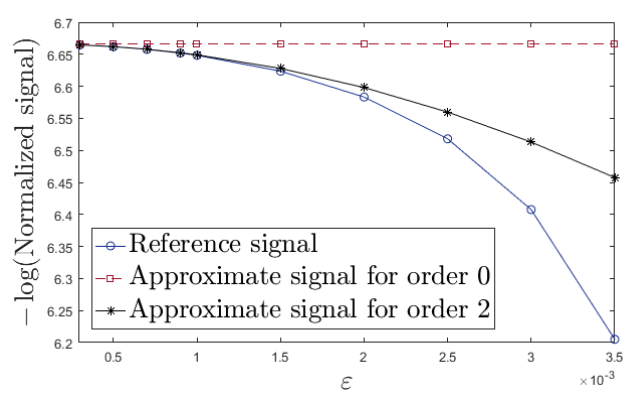

(a) Single circular cell

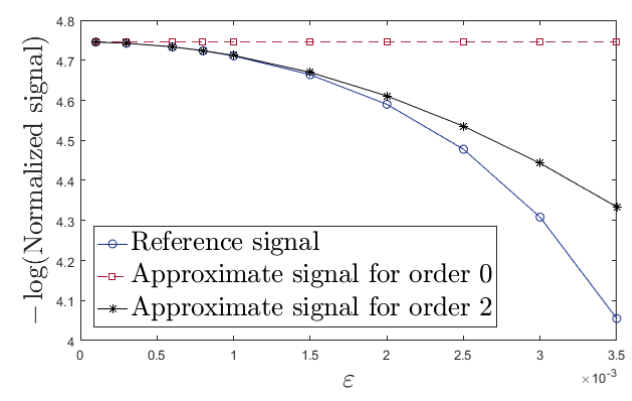

(b) Three circular cells

Fig. 2. Logarithm of normalized signals for two different geometries (see Figure 1) versus $\varepsilon$. The experiment parameters are $\mathcal{D}_{e}=3 \times 10^{-3} \mathrm{~mm}^{2} / \mathrm{s}, b=2500 \mathrm{~s} / \mathrm{mm}^{2}, \boldsymbol{n}=e_{y}, \Delta=5 \mathrm{~ms}$ and $\delta=2.5 \mathrm{~ms}$.

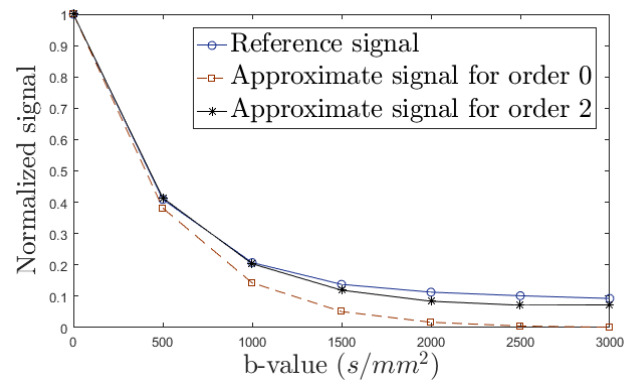

(a)

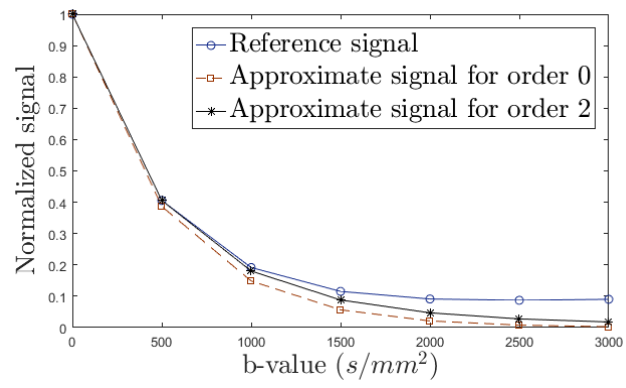

(b)

Fig. 3. Normalized signals versus the $b$-value and two different choices of the parameter $\Delta . \Delta=2.5 \mathrm{~ms}$ for (a) and $\Delta=5 \mathrm{~ms}$ for (b). The geometry of the periodicity box correspond with Figure 1(b). The experiment other parameters are $\mathcal{D}_{e}=3 \times 10^{-3} \mathrm{~mm}^{2} / \mathrm{s}, \varepsilon=6 \times 10^{-3}, \boldsymbol{n}=e_{y}$ and $\delta=2.5 \mathrm{~ms}$. 


\subsection{Examples mimicking membrane effects}

Our model also applies to the heterogeneous case where the diffusion tensor is piece-wise constant and there is no barrier. Formally, it corresponds to replacing $Y_{e}$ by $Y$ in equations (8) and (12)(16), removing the boundary conditions on $\Gamma_{m}$ and considering a piece-wise constant diffusion tensor $\mathcal{D}$ defined on the whole box $Y$.

We use this type of configuration to model a three-compartment configuration with extracellular domain, intra-cellular domain and a membrane domain. The membrane domain corresponds to a thin layer around the intra-cellular domain. As test examples, we consider two different configurations that are depicted in Figure 4 as well as associated finite-element mesh that was used to compute the homogenized tensors. The thickness of the layer in the unit box $Y$ (1 $\mathrm{mm}^{2}$ ) is $L_{0}=0.01 \mathrm{~mm}$. Outside the circles, the intrinsic diffusion is $\mathcal{D}_{e}=3 \times 10^{-3} \mathrm{~mm}^{2} / \mathrm{s}$, inside the circles $\mathcal{D}_{c}=1.7 \times 10^{-3} \mathrm{~mm}^{2} / \mathrm{s}$ and inside the membrane layer $\mathcal{D}_{\text {layer }}=1 \times 10^{-5} \mathrm{~mm}^{2} / \mathrm{s}$.

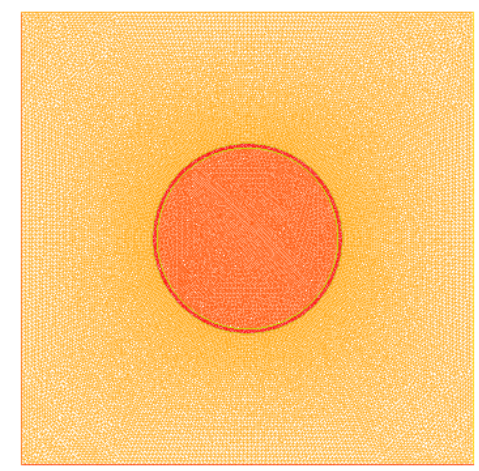

(a)

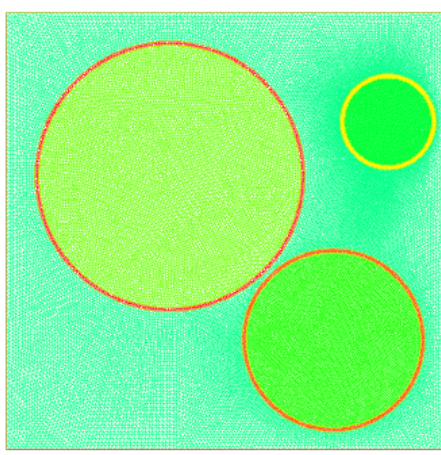

(b)

Fig. 4. Periodicity unit box $Y\left(1 \mathrm{~mm}^{2}\right)$ and associated finite-element mesh for the two different configurations of permeable cells.

Figure 5 displays, for each geometry in Figure 4, the signal according to $b$-value for the three signals $\widetilde{S_{0}}, \widetilde{S_{\varepsilon}}$ and $S_{\varepsilon}^{B T}$ for different values of the parameter $\Delta=5$ and $15 \mathrm{~ms}$. This test shows the same type of results as in the case of impermeable cells. It confirms that the second-order model provides better fit between the "exact signal" and the approximate model.

\section{Conclusion}

In this work we have used homogenization theory to derive a higher-order diffusion tensors for dMRI signals. We obtain numerically computable expressions for these tensors in terms of the microscopic geometry and the signal time profile. Our model has similar structure as the one used in diffusion kurtosis imaging. The numerical results on toy configurations with realistic values for the physical parameters show the superiority of the model over the zeroth-order homogenized problem. In a future work, we shall extend this approach to the case of biological cells with membrane barriers where the homogenized model is much more complex and would couple "double exponential models" with diffusion kurtosis imaging.

\section{A Appendix}

We here give some details of the calculations that lead to the macroscopic equations for $\widehat{\boldsymbol{M}}_{0}, \widehat{\boldsymbol{M}}_{1}$ and $\widehat{\boldsymbol{M}}_{2}$ as well as some symmetry properties for the homogenized tensors. The convention of 


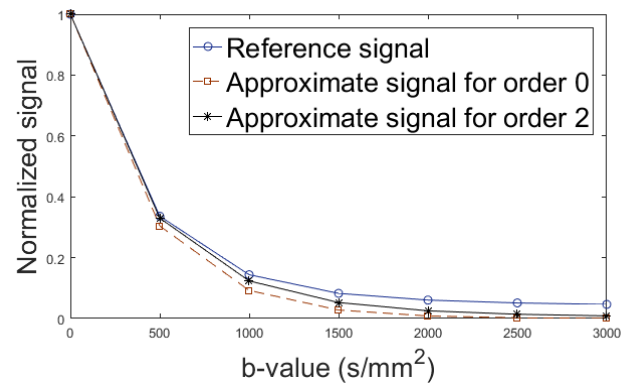

(a) $\Delta=5 \mathrm{~ms}$

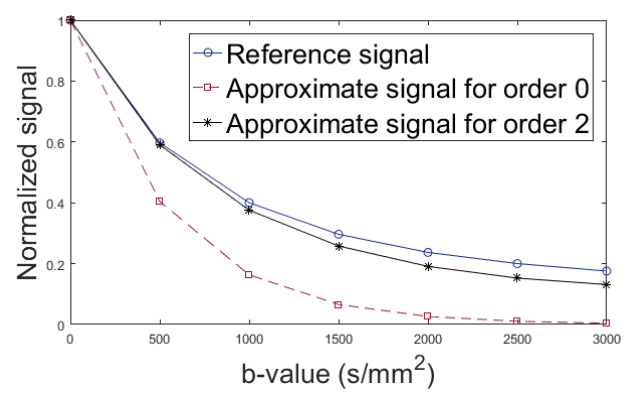

(c) $\Delta=5 \mathrm{~ms}$

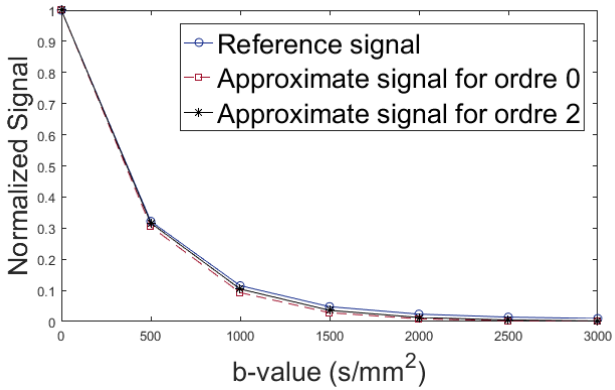

(b) $\Delta=15 \mathrm{~ms}$

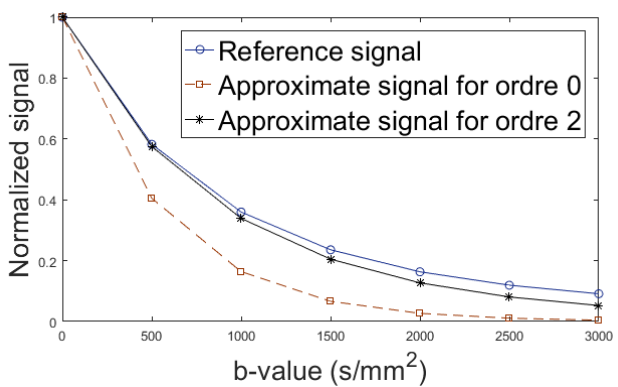

(d) $\Delta=15 \mathrm{~ms}$

Fig. 5. Normalized signals versus the $b$-value for two different geometries correspond with Figure 4. Two different choices of the parameter $\Delta=5 \mathrm{~ms}$ and $15 \mathrm{~ms}$. The experiment other parameters are $\mathcal{D}_{e}=$ $3 \times 10^{-3} \mathrm{~mm}^{2} / \mathrm{s}, \mathcal{D}_{c}=1.7 \times 10^{-3} \mathrm{~mm}^{2} / s, \mathcal{D}_{\text {layer }}=1 \times 10^{-5} \mathrm{~mm}^{2} / \mathrm{s}, L_{0}=0.01 \mathrm{~mm}, \varepsilon=6 \times 10^{-3}, \boldsymbol{n}=e_{y}$ and $\delta=2.5 \mathrm{~ms}$.

summation on repeated indices is adopted in the following expressions. Using the differentiation rules we observe that

$$
\nabla \boldsymbol{M}_{n}\left(\boldsymbol{x}, \frac{\boldsymbol{x}}{\varepsilon}, t\right)=\nabla_{\boldsymbol{x}} \boldsymbol{M}_{n}\left(\boldsymbol{x}, \frac{\boldsymbol{x}}{\varepsilon}, t\right)+\varepsilon^{-1} \nabla_{\boldsymbol{y}} \boldsymbol{M}_{n}\left(\boldsymbol{x}, \frac{\boldsymbol{x}}{\varepsilon}, t\right),
$$

and

$$
\begin{aligned}
\operatorname{div}\left(\mathcal{D} \nabla \boldsymbol{M}_{n}\right)= & \operatorname{div}_{\boldsymbol{x}}\left(\mathcal{D} \nabla_{\boldsymbol{x}} \boldsymbol{M}_{n}\right)+\varepsilon^{-1} \operatorname{div}_{\boldsymbol{x}}\left(\mathcal{D} \nabla_{\boldsymbol{y}} \boldsymbol{M}_{n}\right)+\varepsilon^{-1} \operatorname{div}_{\boldsymbol{y}}\left(\mathcal{D} \nabla_{\boldsymbol{x}} \boldsymbol{M}_{n}\right) \\
& \left.+\varepsilon^{-2} \operatorname{div}_{\boldsymbol{y}} \mathcal{D} \nabla_{\boldsymbol{y}} \boldsymbol{M}_{n}\right) .
\end{aligned}
$$

Substituting $\boldsymbol{M}_{\varepsilon}$ by the asymptotic expansion (7) in problem (6) and multiplying the first two equations by $\varepsilon^{2}$, we then obtain

$$
\left\{\begin{array}{l}
\sum_{n=0}^{+\infty} \varepsilon^{n+2} \frac{\partial}{\partial t} \boldsymbol{M}_{n}+\iota Q f(t) \sum_{n=0}^{+\infty} \varepsilon^{n+2} \boldsymbol{M}_{n}-\sum_{n=0}^{+\infty} \varepsilon^{n+2} \operatorname{div}_{\boldsymbol{x}}\left(\mathcal{D} \nabla_{\boldsymbol{x}} \boldsymbol{M}_{n}\right) \\
\quad-\sum_{n=0}^{+\infty} \varepsilon^{n+1}\left(\operatorname{div}_{\boldsymbol{x}}\left(\mathcal{D} \nabla_{\boldsymbol{y}} \boldsymbol{M}_{n}\right)+\operatorname{div}_{\boldsymbol{y}}\left(\mathcal{D} \nabla_{\boldsymbol{x}} \boldsymbol{M}_{n}\right)\right)-\sum_{n=0}^{+\infty} \varepsilon^{n} \operatorname{div}_{\boldsymbol{y}}\left(\mathcal{D} \nabla_{\boldsymbol{y}} \boldsymbol{M}_{n}\right)=0
\end{array}\right.
$$

that holds for $(\boldsymbol{x}, \boldsymbol{y})$ in $\Omega \times Y_{e}$ and

$$
\left\{\begin{array}{c}
\varepsilon^{0} \mathcal{D} \nabla_{\boldsymbol{y}} \boldsymbol{M}_{0} \cdot \boldsymbol{\nu}+\varepsilon^{1} \mathcal{D}\left(\nabla_{\boldsymbol{y}} \boldsymbol{M}_{1} \cdot \boldsymbol{\nu}+\nabla_{\boldsymbol{x}} \boldsymbol{M}_{0} \cdot \boldsymbol{\nu}\right)+\varepsilon^{2} \mathcal{D}\left(\nabla_{\boldsymbol{y}} \boldsymbol{M}_{2} \cdot \boldsymbol{\nu}+\nabla_{\boldsymbol{x}} \boldsymbol{M}_{1} \cdot \boldsymbol{\nu}\right) \\
\quad+\sum_{n=0}^{+\infty} \varepsilon^{n+1} \mathcal{D}\left(\nabla_{\boldsymbol{y}} \boldsymbol{M}_{n+1} \cdot \boldsymbol{\nu}+\nabla_{\boldsymbol{x}} \boldsymbol{M}_{n} \cdot \boldsymbol{\nu}\right)=0
\end{array}\right.
$$

that holds for $(\boldsymbol{x}, \boldsymbol{y})$ in $\Omega \times \Gamma_{m}$ and finally the initial conditions

$$
\boldsymbol{M}_{0}(\boldsymbol{x}, \boldsymbol{y}, 0)=\boldsymbol{M}_{\mathrm{init}}(\boldsymbol{x}) \quad \text { and } \quad \boldsymbol{M}_{n}(\boldsymbol{x}, \boldsymbol{y}, 0)=0 \text { for } n>0 .
$$


The derivation of averaged equations relies on writing the compatibility conditions for problems of the form

$$
\begin{cases}-\operatorname{div}_{\boldsymbol{y}}\left(\mathcal{D}(\boldsymbol{y}) \nabla_{\boldsymbol{y}} u(\boldsymbol{y})\right)=g(\boldsymbol{y}), & \text { in } Y_{e}, \\ \mathcal{D}(\boldsymbol{y}) \nabla_{\boldsymbol{y}} u(\boldsymbol{y}) \cdot \boldsymbol{\nu}=h(\boldsymbol{y}), & \text { on } \Gamma_{m}, \\ u(\boldsymbol{y}) \text { is } Y \text {-periodic. } & \end{cases}
$$

for some regular data $g$ and $h$. If problem (29) admits a solution (up to an additive constant) then

$$
\int_{Y_{e}} g(\boldsymbol{y}) \mathrm{d} \boldsymbol{y}+\int_{\Gamma_{m}} h(\boldsymbol{y}) \mathrm{d} s(\boldsymbol{y})=0 .
$$

\section{A.1 Macroscopic model for $\widehat{M}_{0}$}

By equating the zeroth-order terms in $\varepsilon$ we get

$$
\begin{cases}-\operatorname{div}_{\boldsymbol{y}}\left(\mathcal{D} \nabla_{\boldsymbol{y}} \boldsymbol{M}_{0}\right)=0, & \text { in } Y_{e}, \\ \mathcal{D} \nabla_{\boldsymbol{y}} \boldsymbol{M}_{0} \cdot \boldsymbol{\nu}=0, & \text { on } \Gamma_{m}, \\ \boldsymbol{M}_{0} \text { is } Y \text {-periodic. } & \end{cases}
$$

We then get that $\boldsymbol{M}_{0}$ does not depend on $\boldsymbol{y}$ and therefore

$$
M_{0}=\widehat{M}_{0} .
$$

By equating the first-order terms in $\varepsilon$ we obtain

$$
\begin{cases}-\operatorname{div}_{\boldsymbol{y}}\left(\mathcal{D} \nabla_{\boldsymbol{y}} \boldsymbol{M}_{1}+\mathcal{D} \nabla_{\boldsymbol{x}} \widehat{\boldsymbol{M}}_{0}\right)=0, & \text { in } Y_{e} \\ \mathcal{D}\left(\nabla_{\boldsymbol{y}} \boldsymbol{M}_{1}+\nabla_{\boldsymbol{x}} \boldsymbol{M}_{0}\right) \cdot \boldsymbol{\nu}=0, & \text { on } \Gamma_{m}, \\ \boldsymbol{M}_{1} \text { is } Y \text {-periodic. } & \end{cases}
$$

The compatibility condition (30) is automatically verified which guarantees a unique (up to an additive constant) solution $\boldsymbol{M}_{1}$ that depends on $\nabla_{\boldsymbol{x}} \widehat{\boldsymbol{M}}_{0}$. One can express the solution to (32) as

$$
\boldsymbol{M}_{1}=\omega \cdot \nabla_{\boldsymbol{x}} \widehat{\boldsymbol{M}}_{0}+\widehat{\boldsymbol{M}}_{1}, \quad \text { in } Y_{e} .
$$

Now, by equating the second-order terms in $\varepsilon$ we get

$$
\begin{cases}-\operatorname{div}_{\boldsymbol{y}}\left(\mathcal{D} \nabla_{\boldsymbol{y}} \boldsymbol{M}_{2}+\mathcal{D} \nabla_{\boldsymbol{x}} \boldsymbol{M}_{1}\right)= & \\ \quad \operatorname{div}_{\boldsymbol{x}}\left(\mathcal{D} \nabla_{\boldsymbol{y}} \boldsymbol{M}_{1}+\mathcal{D} \nabla_{\boldsymbol{x}} \widehat{\boldsymbol{M}}_{0}\right)-\frac{\partial}{\partial t} \widehat{\boldsymbol{M}}_{0}-\iota Q f(t) \widehat{\boldsymbol{M}}_{0}, & \text { in } Y_{e}, \\ \mathcal{D} \nabla_{\boldsymbol{y}} \boldsymbol{M}_{2} \cdot \boldsymbol{\nu}+\mathcal{D} \nabla_{\boldsymbol{x}} \boldsymbol{M}_{1} \cdot \boldsymbol{\nu}=0, & \text { on } \Gamma_{m}, \\ \boldsymbol{M}_{2} \text { is } Y \text {-periodic. } & \end{cases}
$$

The compatibility condition (30) for this problem implies

$$
\int_{Y_{e}}\left(\operatorname{div}_{\boldsymbol{x}}\left(\mathcal{D} \nabla_{\boldsymbol{y}} \boldsymbol{M}_{1}+\mathcal{D} \nabla_{\boldsymbol{x}} \widehat{\boldsymbol{M}}_{0}\right)-\frac{\partial}{\partial t} \widehat{\boldsymbol{M}}_{0}-\iota Q f(t) \widehat{\boldsymbol{M}}_{0}\right) \mathrm{d} \boldsymbol{y}=0 .
$$

Substituting $\boldsymbol{M}_{1}$ by its expression (33) we obtain the macroscopic problem (9) satisfied by $\widehat{\boldsymbol{M}_{0}}$.

\section{A.2 Macroscopic model for $\widehat{M}_{1}$}

To derive the macroscopic model for $\widehat{\boldsymbol{M}}_{1}$ we need to have an expression of $\boldsymbol{M}_{2}$ in terms of $\widehat{\boldsymbol{M}}_{0}$ and $\widehat{\boldsymbol{M}}_{1}$ by solving (34). To simplify the calculations, problem (34) is divided into well-posed sub-systems such that

$$
M_{2}=M_{2}^{1}+M_{2}^{2}+M_{2}^{3}+\widehat{M}_{2},
$$

where the different terms are defined as follows. 
$-\boldsymbol{M}_{2}^{1}$ is the solution of:

$$
\begin{cases}-\operatorname{div}_{\boldsymbol{y}}\left(\mathcal{D} \nabla_{\boldsymbol{y}} \boldsymbol{M}_{2}^{1}\right)=\operatorname{div}_{\boldsymbol{y}}\left(\mathcal{D} \nabla_{\boldsymbol{x}} \widehat{\boldsymbol{M}}_{1}\right), & \text { in } Y_{e}, \\ \mathcal{D} \nabla_{\boldsymbol{y}} \boldsymbol{M}_{2}^{1} \cdot \boldsymbol{\nu}+\mathcal{D} \nabla_{\boldsymbol{x}} \widehat{\boldsymbol{M}}_{1} \cdot \boldsymbol{\nu}=0, & \text { on } \Gamma_{m}, \\ \boldsymbol{M}_{2}^{1} \text { is } Y \text {-periodic. } & \end{cases}
$$

Thus, using for $i=1, \ldots, d$ the cell problems (8), which are well posed according to (30), one can express the solution as

$$
\boldsymbol{M}_{2}^{1}=\omega \cdot \nabla_{\boldsymbol{x}} \widehat{\boldsymbol{M}}_{1}+\widehat{\boldsymbol{M}}_{2}^{1}, \quad \text { in } Y_{e} .
$$

$-\boldsymbol{M}_{2}^{2}$ is the solution of:

$$
\begin{cases}-\operatorname{div}_{\boldsymbol{y}}\left(\mathcal{D} \nabla_{\boldsymbol{y}} \boldsymbol{M}_{2}^{2}\right)=\operatorname{div} \boldsymbol{y}\left(\mathcal{D} \nabla_{\boldsymbol{x}}\left(\omega \cdot \nabla_{\boldsymbol{x}} \widehat{\boldsymbol{M}}_{0}\right)\right), & \text { in } Y_{e}, \\ \mathcal{D} \nabla_{\boldsymbol{y}} \boldsymbol{M}_{2}^{2} \cdot \boldsymbol{\nu}+\mathcal{D} \nabla_{\boldsymbol{x}}\left(\omega \cdot \nabla_{\boldsymbol{x}} \widehat{\boldsymbol{M}}_{0}\right) \cdot \boldsymbol{\nu}=0, & \text { on } \Gamma_{m}, \\ \boldsymbol{M}_{2}^{2} \text { is } Y \text {-periodic. } & \end{cases}
$$

For $i, j=1, \ldots, d$, the cell problems (15) are well posed according to the compatibility condition (30). Therefore, one can express the solution as

$$
\boldsymbol{M}_{2}^{2}=\left(\nabla_{\boldsymbol{x}}^{2} \boldsymbol{M}_{0}\right)_{i j} \chi_{i j}+\widehat{\boldsymbol{M}}_{2}^{2}, \quad \text { in } Y_{e} .
$$

Observe that, since $\left\langle\chi_{i j}\right\rangle_{Y_{e}}=0$, we have $\widehat{\boldsymbol{M}}_{2}^{2}=\left\langle\boldsymbol{M}_{2}^{2}\right\rangle_{Y_{e}}$.

$-\boldsymbol{M}_{2}^{3}$ is the solution of:

$$
\begin{cases}-\operatorname{div}_{\boldsymbol{y}}\left(\mathcal{D} \nabla_{\boldsymbol{y}} \boldsymbol{M}_{2}^{3}\right)=\operatorname{div}_{\boldsymbol{x}}\left(\mathcal{D} \nabla_{\boldsymbol{y}} \boldsymbol{M}_{1}+\mathcal{D} \nabla_{\boldsymbol{x}} \widehat{\boldsymbol{M}}_{0}\right)-\frac{\partial}{\partial t} \widehat{\boldsymbol{M}}_{0}-\iota Q f(t) \widehat{\boldsymbol{M}}_{0}, & \text { in } Y_{e} \\ \mathcal{D} \nabla_{\boldsymbol{y}} \boldsymbol{M}_{2}^{3} \cdot \boldsymbol{\nu}=0, & \text { on } \Gamma_{m} \\ \boldsymbol{M}_{2}^{3} \text { is } Y \text {-periodic. } & \end{cases}
$$

Let us define

$$
F:=\operatorname{div}_{\boldsymbol{x}}\left(\mathcal{D} \nabla_{\boldsymbol{y}} \boldsymbol{M}_{1}+\mathcal{D} \nabla_{\boldsymbol{x}} \widehat{\boldsymbol{M}}_{0}\right)-\frac{\partial}{\partial t} \widehat{\boldsymbol{M}}_{0}-\iota Q f(t) \widehat{\boldsymbol{M}}_{0} .
$$

Then, according to the macroscopic model (9) for $\boldsymbol{M}_{0}$, we remark that

$$
\langle F\rangle_{Y_{e}}:=\operatorname{div}_{\boldsymbol{x}}\left(\mathbb{D} \nabla_{\boldsymbol{x}} \widehat{\boldsymbol{M}}_{0}\right)-\frac{\partial}{\partial t} \widehat{\boldsymbol{M}}_{0}-\iota Q f(t) \widehat{\boldsymbol{M}}_{0}=0 .
$$

Then, we have

$$
F=F-\langle F\rangle_{Y_{e}}=\left(\nabla_{\boldsymbol{x}}^{2} \widehat{\boldsymbol{M}}_{0}\right)_{i j}\left(\mathcal{D} \frac{\partial}{\partial y_{i}} \omega_{j}+\mathcal{D} \delta_{i j}-\mathbb{D}_{i j}\right) .
$$

We consider, for $i, j=1, \ldots, d$, the cell problems (15). The compatibility condition (30) guarantees a unique (up to an additive constant) solution $\boldsymbol{M}_{2}^{3}$ that depends on $\nabla_{\boldsymbol{x}} \nabla_{\boldsymbol{x}} \boldsymbol{M}_{0}$ through

$$
\boldsymbol{M}_{2}^{3}=\left(\nabla_{\boldsymbol{x}}^{2} \widehat{\boldsymbol{M}}_{0}\right)_{i j} \varphi_{i j}+\widehat{\boldsymbol{M}}_{2}^{3} \quad \text { in } Y_{e} .
$$

By regrouping the expressions of $\boldsymbol{M}_{2}^{1}, \boldsymbol{M}_{2}^{2}$ and $\boldsymbol{M}_{2}^{3}$ we obtain the expression for $\boldsymbol{M}_{2}$

$$
\boldsymbol{M}_{2}=\omega \cdot \nabla_{\boldsymbol{x}} \widehat{\boldsymbol{M}}_{1}+\left(\nabla_{\boldsymbol{x}}^{2} \widehat{\boldsymbol{M}}_{0}\right)_{i j}\left(\chi_{i j}+\varphi_{i j}\right)+\widehat{\boldsymbol{M}}_{2} \quad \text { in } Y_{e} .
$$

By equating the third-order terms in $\varepsilon$ we get

$$
\begin{cases}-\operatorname{div}_{\boldsymbol{y}}\left(\mathcal{D} \nabla_{\boldsymbol{y}} \boldsymbol{M}_{3}+\mathcal{D} \nabla_{\boldsymbol{x}} \boldsymbol{M}_{2}\right)= & \\ \quad \operatorname{div}_{\boldsymbol{x}}\left(\mathcal{D} \nabla_{\boldsymbol{y}} \boldsymbol{M}_{2}+\mathcal{D} \nabla_{\boldsymbol{x}} \boldsymbol{M}_{1}\right)-\frac{\partial}{\partial t} \boldsymbol{M}_{1}-\iota Q f(t) \boldsymbol{M}_{1}, & \text { in } Y_{e}, \\ \mathcal{D} \nabla_{\boldsymbol{y}} \boldsymbol{M}_{3} \cdot \boldsymbol{\nu}+\mathcal{D} \nabla_{\boldsymbol{x}} \boldsymbol{M}_{2} \cdot \boldsymbol{\nu}=0, & \text { on } \Gamma_{m} \\ \boldsymbol{M}_{3} \text { is } Y \text {-periodic. } & \end{cases}
$$


The use of the compatibility condition on $\boldsymbol{M}_{3}$ for this problem implies

$$
\int_{Y_{e}}\left(\operatorname{div}_{\boldsymbol{x}}\left(\mathcal{D}\left(\nabla_{\boldsymbol{y}} \boldsymbol{M}_{2}+\nabla_{\boldsymbol{x}} \boldsymbol{M}_{1}\right)\right)-\frac{\partial}{\partial t} \boldsymbol{M}_{1}-\iota Q f(t) \boldsymbol{M}_{1}\right) \mathrm{d} \boldsymbol{y}=0 .
$$

By substituting the expressions for $\boldsymbol{M}_{1}$ and $\boldsymbol{M}_{2}$ and making some recollections we obtain the following macroscopic model for $\widehat{\boldsymbol{M}}_{1}$.

$$
\begin{cases}\frac{\partial}{\partial t} \widehat{\boldsymbol{M}}_{1}+\iota Q f(t) \widehat{\boldsymbol{M}}_{1}-\operatorname{div}_{\boldsymbol{x}}\left(\mathbb{D} \nabla_{\boldsymbol{x}} \widehat{\boldsymbol{M}}_{1}\right)=\operatorname{div}_{\boldsymbol{x}}\left(\mathbb{H}: \nabla_{\boldsymbol{x}}^{2} \boldsymbol{M}_{0}\right), & \text { in } \Omega \times] 0, T[ \\ \widehat{\boldsymbol{M}}_{1}(\cdot, 0)=\mathbf{0}, & \text { in } \Omega .\end{cases}
$$

where we have defined the homogenized third-order tensor $\mathbb{H}$ through

$$
\mathbb{H}_{i j k}:=\left\langle\mathcal{D} \frac{\partial}{\partial y_{k}}\left(\chi_{i j}+\varphi_{i j}\right)\right\rangle_{Y_{e}}, \quad i, j, k=1, \ldots, d .
$$

Since $\mathbb{H}$ is antisymmetric with respect to its last two arguments, i.e., $\mathbb{H}_{i j k}=-\mathbb{H}_{i k j}$ for $i, j, k$ $\in\{1, \ldots, d\}$ (see [23] and also Section A.4), one can easily check that $\operatorname{div}_{\boldsymbol{x}}\left(\mathbb{H}: \nabla_{\boldsymbol{x}}^{2} \boldsymbol{M}_{0}\right)=0$. Therefore, the macroscopic equations (37) simplify to (10).

\section{A.3 Macroscopic model for $\widehat{M}_{2}$}

With the same reasoning as above, to derive the macroscopic model for $\widehat{\boldsymbol{M}_{2}}$, we first need to have an expression of $\boldsymbol{M}_{3}$ in terms of $\widehat{\boldsymbol{M}}_{0}, \widehat{\boldsymbol{M}}_{1}$ and $\widehat{\boldsymbol{M}_{2}}$. We consider problem (36) and decompose its solution $M_{3}$ as

$$
M_{3}=M_{3}^{1}+M_{3}^{2}+M_{3}^{3}+M_{3}^{4}+\widehat{M}_{3},
$$

where the different terms are defined as follows.

$-M_{3}^{1}$ is the solution of:

$$
\begin{cases}-\operatorname{div}_{\boldsymbol{y}}\left(\mathcal{D} \nabla_{\boldsymbol{y}} \boldsymbol{M}_{3}^{1}\right)=\operatorname{div}_{\boldsymbol{y}}\left(\mathcal{D} \nabla_{\boldsymbol{x}} \widehat{\boldsymbol{M}}_{2}\right), & \text { in } Y_{c} \\ \mathcal{D} \nabla_{\boldsymbol{y}} \boldsymbol{M}_{3}^{1} \cdot \boldsymbol{\nu}+\mathcal{D} \nabla_{\boldsymbol{x}} \widehat{\boldsymbol{M}}_{2} \cdot \boldsymbol{\nu}=0, & \text { on } \Gamma_{m}, \\ \boldsymbol{M}_{3}^{1} \text { is } Y \text {-periodic. } & \end{cases}
$$

Using for $i=1, \ldots, d$ the cell problems (8) which are well posed according (30), one can express the solution as

$$
\boldsymbol{M}_{3}^{1}=\omega \cdot \nabla_{\boldsymbol{x}} \widehat{\boldsymbol{M}}_{2}+\widehat{\boldsymbol{M}}_{3}^{1} \quad \text { in } Y_{e}
$$

$-\boldsymbol{M}_{3}^{2}$ is the solution of:

$$
\begin{cases}-\operatorname{div}_{\boldsymbol{y}}\left(\mathcal{D} \nabla_{\boldsymbol{y}} \boldsymbol{M}_{3}^{2}\right)=\operatorname{div}_{\boldsymbol{y}}\left(\mathcal{D} \nabla_{\boldsymbol{x}} \boldsymbol{M}_{2}^{1}\right), & \text { in } Y_{e} \\ \mathcal{D} \nabla_{\boldsymbol{y}} \boldsymbol{M}_{3}^{2} \cdot \boldsymbol{\nu}+\mathcal{D} \nabla_{\boldsymbol{x}} \boldsymbol{M}_{2}^{1} \cdot \boldsymbol{\nu}=0, & \text { on } \Gamma_{m} \\ \boldsymbol{M}_{3}^{2} \text { is } Y \text {-periodic. } & \end{cases}
$$

Since we have

$$
\operatorname{div}_{\boldsymbol{y}}\left(\mathcal{D} \nabla_{\boldsymbol{x}} \boldsymbol{M}_{2}^{1}\right)=\left(\nabla_{\boldsymbol{x}}^{2} \widehat{\boldsymbol{M}}_{1}\right)_{i j} \frac{\partial}{\partial y_{i}}\left(\mathcal{D} \omega_{j}\right),
$$

the solution can be expressed as

$$
\boldsymbol{M}_{3}^{2}=\left(\nabla_{\boldsymbol{x}}^{2} \widehat{\boldsymbol{M}}_{1}\right)_{i j} \chi_{i j}+\widehat{\boldsymbol{M}}_{3}^{2}, \quad \text { in } Y_{e} .
$$


$-\boldsymbol{M}_{3}^{3}$ is the solution of:

$$
\begin{cases}-\operatorname{div}_{\boldsymbol{y}}\left(\mathcal{D} \nabla_{\boldsymbol{y}} \boldsymbol{M}_{3}^{3}\right)=\operatorname{div}_{\boldsymbol{y}}\left(\mathcal{D} \nabla_{\boldsymbol{x}}\left(\boldsymbol{M}_{2}^{2}+\boldsymbol{M}_{2}^{3}\right)\right), & \text { in } Y_{e}, \\ \mathcal{D} \nabla_{\boldsymbol{y}} \boldsymbol{M}_{3}^{3} \cdot \boldsymbol{\nu}+\mathcal{D} \nabla_{\boldsymbol{x}}\left(\boldsymbol{M}_{2}^{2}+\boldsymbol{M}_{2}^{3}\right) \cdot \boldsymbol{\nu}=0, & \text { on } \Gamma_{m}, \\ \boldsymbol{M}_{3}^{3} \text { is } Y \text {-periodic. } & \end{cases}
$$

For $i, j, k=1, \ldots, d$, we consider the cell problems (14) that allow us to express the solution as

$$
\boldsymbol{M}_{3}^{3}=\left(\nabla_{\boldsymbol{x}}^{3} \widehat{\boldsymbol{M}}_{0}\right)_{i j k} \mathcal{R}_{i j k}+\widehat{\boldsymbol{M}}_{3}^{3}, \quad \text { in } Y_{e} .
$$

$-\boldsymbol{M}_{3}^{4}$ is the solution of:

$$
\begin{cases}-\operatorname{div}_{\boldsymbol{y}}\left(\mathcal{D} \nabla_{\boldsymbol{y}} \boldsymbol{M}_{3}^{4}\right)=\operatorname{div}_{\boldsymbol{x}}\left(\mathcal{D} \nabla_{\boldsymbol{y}} \boldsymbol{M}_{2}+\mathcal{D} \nabla_{\boldsymbol{x}} \boldsymbol{M}_{1}\right)-\frac{\partial}{\partial t} \boldsymbol{M}_{1}-\iota Q f(t) \boldsymbol{M}_{1}, & \text { in } Y_{e} \\ \mathcal{D} \nabla_{\boldsymbol{y}} \boldsymbol{M}_{3}^{4} \cdot \boldsymbol{\nu}=0, & \text { on } \Gamma_{m} \\ \boldsymbol{M}_{3}^{4} \text { is } Y \text {-periodic. } & \end{cases}
$$

Let us define

$$
G:=\operatorname{div}_{\boldsymbol{x}}\left(\mathcal{D} \nabla_{\boldsymbol{y}} \boldsymbol{M}_{2}+\mathcal{D} \nabla_{\boldsymbol{x}} \boldsymbol{M}_{1}\right)-\frac{\partial}{\partial t} \boldsymbol{M}_{1}-\iota Q f(t) \boldsymbol{M}_{1} .
$$

According to the macroscopic model (10) for $\widehat{\boldsymbol{M}}_{2}$, we remark that

$$
\langle G\rangle_{Y_{e}}=-\frac{\partial}{\partial t} \widehat{\boldsymbol{M}}_{1}-\iota Q f(t) \widehat{\boldsymbol{M}}_{1}+\operatorname{div}_{\boldsymbol{x}}\left(\mathbb{D} \nabla_{\boldsymbol{x}} \widehat{\boldsymbol{M}}_{1}\right)=0 .
$$

Then, we have

$$
\begin{aligned}
G= & G-\langle G\rangle_{Y_{e}}=\operatorname{div}_{\boldsymbol{x}}\left(\mathcal{D} \nabla_{\boldsymbol{y}} \boldsymbol{M}_{2}+\mathcal{D} \nabla_{\boldsymbol{x}} \boldsymbol{M}_{1}\right)-\operatorname{div}_{\boldsymbol{x}}\left(\left\langle\mathcal{D} \nabla_{\boldsymbol{y}} \boldsymbol{M}_{2}+\mathcal{D} \nabla_{\boldsymbol{x}} \boldsymbol{M}_{1}\right\rangle_{Y_{e}}\right) \\
& -\omega_{i} \frac{\partial}{\partial t}\left(\nabla_{\boldsymbol{x}} \widehat{\boldsymbol{M}}_{0}\right)_{i}-\iota Q f(t) \omega \cdot \nabla_{\boldsymbol{x}} \widehat{\boldsymbol{M}}_{0} .
\end{aligned}
$$

Using (9) and that $\frac{\partial}{\partial x_{i}}\left(Q \boldsymbol{M}_{0}\right)=Q \frac{\partial}{\partial x_{i}} \widehat{\boldsymbol{M}}_{0}+q_{i} \widehat{\boldsymbol{M}}_{0}$, for each $i$ we get

$$
\frac{\partial}{\partial t}\left(\nabla_{\boldsymbol{x}} \widehat{\boldsymbol{M}}_{0}\right)_{i}+\iota Q f(t)\left(\nabla_{\boldsymbol{x}} \widehat{\boldsymbol{M}}_{0}\right)_{i}=\left(\nabla_{\boldsymbol{x}} \operatorname{div}_{\boldsymbol{x}}\left(\mathbb{D} \nabla_{\boldsymbol{x}} \widehat{\boldsymbol{M}}_{0}\right)\right)_{i}-\iota q_{i} f(t) \widehat{\boldsymbol{M}}_{0}
$$

Substituting the expressions for $\boldsymbol{M}_{1}$ and $\boldsymbol{M}_{2}$ and making some recollection, we obtain

$$
\begin{aligned}
G= & \left(\nabla_{\boldsymbol{x}}^{2} \widehat{\boldsymbol{M}}_{1}\right)_{i j}\left(\mathcal{D} \frac{\partial}{\partial y_{i}} \omega_{j}+\mathcal{D} \delta_{i j}-\mathbb{D}_{i j}\right)+\left(\nabla_{\boldsymbol{x}}^{3} \widehat{\boldsymbol{M}}_{0}\right)_{i j k}\left(\mathcal{D} \omega_{k} \delta_{i j}+\mathcal{D} \frac{\partial}{\partial y_{i}}\left(\chi_{j k}+\varphi_{j k}\right)-\mathbb{H}_{i j k}\right) \\
& -\omega \cdot \nabla_{\boldsymbol{x}} \operatorname{div}_{\boldsymbol{x}}\left(\mathbb{D} \nabla_{\boldsymbol{x}} \widehat{\boldsymbol{M}}_{0}\right)+\iota \omega \cdot \boldsymbol{q} f(t) \widehat{\boldsymbol{M}}_{0} .
\end{aligned}
$$

We finally can express the solution as

$$
\boldsymbol{M}_{3}^{4}=\left(\nabla_{\boldsymbol{x}}^{2} \widehat{\boldsymbol{M}}_{1}\right): \varphi+\left(\nabla_{\boldsymbol{x}}^{3} \widehat{\boldsymbol{M}}_{0}\right) \therefore \mathcal{Q}-\theta \cdot \nabla_{\boldsymbol{x}} \operatorname{div}_{\boldsymbol{x}}\left(\mathbb{D} \nabla_{\boldsymbol{x}} \widehat{\boldsymbol{M}}_{0}\right)+\iota \theta \cdot \boldsymbol{q} f(t) \widehat{\boldsymbol{M}}_{0}+\widehat{\boldsymbol{M}}_{3}^{4}, \quad \text { in } Y_{e}
$$

where $\widehat{\boldsymbol{M}}_{3}^{4}=\left\langle\boldsymbol{M}_{3}^{4}\right\rangle_{Y_{e}}$ and, for $i, j, k=1, \ldots, d, \theta_{i}$ and $\mathcal{Q}_{i j k}$ are solutions of cell problems (12) and (13), respectively.

Collecting all expressions obtained for $\boldsymbol{M}_{3}^{i}$ one gets the expression of $\boldsymbol{M}_{3}$

$$
\begin{aligned}
\boldsymbol{M}_{3}= & \omega \cdot \nabla_{\boldsymbol{x}} \widehat{\boldsymbol{M}}_{2}+\left(\nabla_{\boldsymbol{x}}^{2} \widehat{\boldsymbol{M}}_{1}\right):(\chi+\varphi)+\left(\nabla_{\boldsymbol{x}}^{3} \widehat{\boldsymbol{M}}_{0}\right) \therefore(\mathcal{R}+\mathcal{Q}) \\
& -\theta \cdot \nabla_{\boldsymbol{x}}\left(\operatorname{div}_{\boldsymbol{x}}\left(\mathbb{D}_{\boldsymbol{x}} \widehat{\boldsymbol{M}}_{0}\right)\right)+\iota \theta \cdot \boldsymbol{q} f(t) \widehat{\boldsymbol{M}}_{0}+\widehat{\boldsymbol{M}}_{3}, \quad \text { in } Y_{e} .
\end{aligned}
$$

The next step is to equate the fourth-order terms in $\varepsilon$ which gives.

$$
\begin{cases}\quad-\operatorname{div}_{\boldsymbol{y}}\left(\mathcal{D} \nabla_{\boldsymbol{y}} \boldsymbol{M}_{4}+\mathcal{D} \nabla_{\boldsymbol{x}} \boldsymbol{M}_{3}\right)= & \\ \quad \operatorname{div}_{\boldsymbol{x}}\left(\mathcal{D} \nabla_{\boldsymbol{y}} \boldsymbol{M}_{3}+\mathcal{D} \nabla_{\boldsymbol{x}} \boldsymbol{M}_{2}\right)-\frac{\partial}{\partial t} \boldsymbol{M}_{2}-\iota Q f(t) \boldsymbol{M}_{2}, & \text { in } Y_{e}, \\ \mathcal{D} \nabla_{\boldsymbol{y}} \boldsymbol{M}_{4} \cdot \boldsymbol{\nu}+\mathcal{D} \nabla_{\boldsymbol{x}} \boldsymbol{M}_{3} \cdot \boldsymbol{\nu}=0, & \text { on } \Gamma_{m}, \\ \boldsymbol{M}_{4} \text { is } Y \text {-periodic. } & \end{cases}
$$


Writing the compatibility condition (30) for this problem

$$
\int_{Y_{e}}\left(\operatorname{div}_{\boldsymbol{x}}\left(\mathcal{D}\left(\nabla_{\boldsymbol{y}} \boldsymbol{M}_{3}+\nabla_{\boldsymbol{x}} \boldsymbol{M}_{2}\right)\right)-\frac{\partial}{\partial t} \boldsymbol{M}_{2}-\iota Q f(t) \boldsymbol{M}_{2}\right) \mathrm{d} \boldsymbol{y}=0 .
$$

Substituting the expressions for $\boldsymbol{M}_{2}$ and $\boldsymbol{M}_{3}$ and after simplification with the use of $\left\langle\omega_{i}\right\rangle_{Y_{e}}=$ $\left\langle\chi_{i j}\right\rangle_{Y_{e}}=\left\langle\varphi_{i j}\right\rangle_{Y_{e}}=0$, we obtain the macroscopic model (11) for $\widehat{\boldsymbol{M}}_{2}$.

\section{A.4 Symmetry properties of $\mathbb{H}$}

By using (8), (15), (16) and the fact that $\left\langle\omega_{i}\right\rangle_{Y_{e}}=0$, the components of the homogenized thirdorder tensor $\mathbb{H}$ can be rewritten as

$$
\begin{aligned}
\mathbb{H}_{i j k} & =\frac{1}{\left|Y_{e}\right|} \int_{Y_{e}} \mathcal{D} \nabla\left(\chi_{i j}+\varphi_{i j}\right) \cdot e_{k} \mathrm{~d} \boldsymbol{y}=-\frac{1}{\left|Y_{e}\right|} \int_{Y_{e}} \mathcal{D} \nabla \omega_{k} \cdot \nabla\left(\chi_{i j}+\varphi_{i j}\right) \mathrm{d} \boldsymbol{y} \\
& =\frac{1}{\left|Y_{e}\right|} \int_{Y_{e}} \operatorname{div}\left(\mathcal{D} \nabla\left(\chi_{i j}+\varphi_{i j}\right)\right) \omega_{k} \mathrm{~d} \boldsymbol{y}-\frac{1}{\left|Y_{e}\right|} \int_{\Gamma_{m}} \mathcal{D} \nabla\left(\chi_{i j}+\varphi_{i j}\right) \cdot \boldsymbol{\nu} \omega_{k} \mathrm{~d} s \\
& =-\frac{2}{\left|Y_{e}\right|} \int_{Y_{e}} \frac{\partial}{\partial y_{i}}\left(\mathcal{D} \omega_{j}\right) \omega_{k} \mathrm{~d} \boldsymbol{y}+\int_{\Gamma_{m}} \mathcal{D} \omega_{j} \cdot \nu_{i} \omega_{k} \mathrm{~d} s .
\end{aligned}
$$

Since $\frac{\partial}{\partial y_{i}}\left(\sigma \omega_{j}\right)=\mathcal{D} \nabla \omega_{j} \cdot e_{i}=\operatorname{div}\left(\mathcal{D} \omega_{j} e_{i}\right)$, using the divergence theorem for the last term we finally get

$$
\mathbb{H}_{i j k}=\left\langle-\mathcal{D} \nabla \omega_{j} \cdot e_{i} \omega_{k}+\mathcal{D} \omega_{j} e_{i} \cdot \nabla \omega_{k}\right\rangle_{Y_{e}} .
$$

This expression clearly shows the anti-symmetry property $\mathbb{H}_{i j k}=-\mathbb{H}_{i k j}$ which when combined with the symmetry of $\nabla^{2} \boldsymbol{M}_{0}$ yields $\operatorname{div}_{\boldsymbol{x}}\left(\mathbb{H}: \nabla_{\boldsymbol{x}}^{2} \widehat{\boldsymbol{M}}_{0}\right)=0$.

\section{A.5 Alternative expression for $\mathbb{T}$}

The expression for the fourth-order tensor can be further simplified. Using (8), (13) and (14) we have

$$
\begin{aligned}
\mathbb{T}_{i j k l} & =\frac{1}{\left|Y_{e}\right|} \int_{Y_{e}} \mathcal{D} \nabla\left(\mathcal{Q}_{i j k}+\mathcal{R}_{i j k}\right) \cdot e_{l} \mathrm{~d} \boldsymbol{y}=-\frac{1}{\left|Y_{e}\right|} \int_{Y_{e}} \mathcal{D} \nabla \omega_{l} \cdot \nabla\left(\mathcal{Q}_{i j k}+\mathcal{R}_{i j k}\right) \mathrm{d} \boldsymbol{y} \\
& =-\frac{2}{\left|Y_{e}\right|} \int_{Y_{e}} \frac{\partial}{\partial y_{i}}\left(\mathcal{D}\left(\chi_{j k}+\varphi_{j k}\right)\right) \omega_{l} \mathrm{~d} \boldsymbol{y}-\frac{1}{\left|Y_{e}\right|} \int_{Y_{e}} \mathcal{D} \omega_{k} \delta_{i j} \omega_{l} \mathrm{~d} \boldsymbol{y} \\
& +\frac{1}{\left|Y_{e}\right|} \int_{\Gamma_{m}} \mathcal{D}\left(\chi_{j k}+\varphi_{j k}\right) \nu_{i} \omega_{l} \mathrm{~d} s \\
& =-\frac{1}{\left|Y_{e}\right|} \int_{Y_{e}} \mathcal{D} \nabla\left(\chi_{j k}+\varphi_{j k}\right) \cdot e_{i} \omega_{l} \mathrm{~d} \boldsymbol{y}-\frac{1}{\left|Y_{e}\right|} \int_{Y_{e}} \operatorname{div}\left(\mathcal{D}\left(\chi_{j k}+\varphi_{j k}\right) e_{i}\right) \omega_{l} \mathrm{~d} \boldsymbol{y} \\
& -\frac{1}{\left|Y_{e}\right|} \int_{Y_{e}} \mathcal{D} \omega_{k} \omega_{l} \delta_{i j} \mathrm{~d} \boldsymbol{y}+\frac{1}{\left|Y_{e}\right|} \int_{\Gamma_{m}} \mathcal{D}\left(\chi_{j k}+\varphi_{j k}\right) \nu_{i} \omega_{l} \mathrm{~d} s .
\end{aligned}
$$

Applying the divergence theorem for the surface integral we end up with

$$
\mathbb{T}_{i j k l}=\left\langle-\mathcal{D} \nabla\left(\chi_{j k}+\varphi_{j k}\right) e_{i} \omega_{l}+\mathcal{D}\left(\chi_{j k}+\varphi_{j k}\right) e_{i} \nabla \omega_{l}-\mathcal{D} \omega_{k} \omega_{l} \delta_{i j}\right\rangle_{Y_{e}}
$$

\section{References}

1. G. Allaire, M. Briane, and M. Vanninathan. A comparison between two-scale asymptotic expansions and Bloch wave expansions for the homogenization of periodic structures. SeMA, 73(3):237-259, 2016.

2. G. Allaire and T. Yamada. Optimization of dispersive coefficients in the homogenization of the wave equation in periodic structures. submitted, 2016. 
3. I. V. Andrianov, V. I. Bolshakov, V. V. Danishevs'kyy, and D. Weichert. Higher order asymptotic homogenization and wave propagation in periodic composite materials. Proc. Roy. Soc. A, 464(2093):1181-1201, 2008.

4. A. Bensoussan, J. L. Lions, and G. Papanicolaou. Asymptotic Analysis for Periodic Structures. AMS Chelsea Publishing, Providence, RI, 2011.

5. P. T. Callaghan, A. Coy, D. MacGowan, K. J. Packer, and F. O. Zelaya. Diffraction-like effects in nmr diffusion studies of fluids in porous solids. Nature, 351(6326):467-469, 1991.

6. Paul T. Callaghan and Janez Stepianik. Frequency-domain analysis of spin motion using modulatedgradient NMR. Journal of Magnetic Resonance, Series A, 117(1):118-122, 1995.

7. J. Chen, W. Liu, H. Zhang, L. Lacy, X. Yang, S.-K. Song, S. A. Wickline, and X. Yu. Regional ventricular wall thickening reflects changes in cardiac fiber and sheet structure during contraction: quantification with diffusion tensor MRI. American Journal of Physiology - Heart and Circulatory Physiology, 289(5):H1898-H1907, 2005.

8. J. Coatléven, H. Haddar, and J.-R. Li. A new macroscopic model including membrane exchange for diffusion MRI. SIAM Journal of Applied Mathematics, 74(2):516-546, 2014.

9. C. Conca, R. Orive, and M. Vanninathan. On Burnett coefficients in periodic media. J. Math. Phys, 3:11, 2006.

10. D. S. Grebenkov. From the microstructure to diffusion MRI, and back. In R. Valiullin, editor, Diffusion NMR of Confined Systems: Fluid Transport in Porous Solids and Heterogeneous Materials, New Developments in NMR, pages 52-110. The Royal Society of Chemistry, 2017.

11. H. Haddar, J.-R. Li, and S. Schiavi. Adapting the Kärger model to account for finite diffusion-encoding pulses in diffusion MRI. IMA Journal of Applied Mathematics, 81(5):779-794, 2016.

12. H. Haddar, J.-R. Li, and S. Schiavi. A macroscopic model for the diffusion MRI signal accounting for time-dependent diffusivity. SIAM Journal on Applied Mathematics, 76(3):930-949, 2016.

13. F. Hecht, O. Pironneau, and J. Morice. Freefem. www.freefem.org.

14. T. Hui and C. Oskay. A high order homogenization model for transient dynamics of heterogeneous media including micro-inertia effects. Comput. Methods Appl. Mech. Engrg., 273:181-203, 2014.

15. J. H. Jensen, J. A. Helpern, A. Ramani, H. Lu, and K. Kaczynski. Diffusional kurtosis imaging: the quantification of non-Gaussian water diffusion by means of magnetic resonance imaging. Magnetic Resonance in Medicine, 53:1432-1440, 2005.

16. D. Le Bihan and H. Johansen-Berg. Diffusion MRI at 25: Exploring brain tissue structure and function. NeuroImage, 61(2):324-341, 2012.

17. J.-R. Li, H. T. Nguyen, D. Van Nguyen, H. Haddar, J. Coatléven, and D. Le Bihan. Numerical study of a macroscopic finite pulse model of the diffusion MRI signal. Journal of Magnetic Resonance, 248:54-65, 2014.

18. C. Liu, R. Bammer, B. Acar, and M. E. Moseley. Characterizing non-Gaussian diffusion by using generalized diffusion tensors. Magnetic Resonance in Medicine, 51:924-937, 2004.

19. H. Lu, J. H. Jensen, A. Ramani, and J. A. Helpern. Three dimensional characterization of non Gaussian water diffusion in humans using diffusion kurtosis imaging. NMR Biomed., 19:236-247, 2006.

20. M. Peerlings M. Ameen and R.H.J. Geers. Higher-order asymptotic homogenization of periodic linear elastic composite materials at low scale separation. Contributions to the Foundations of Multidisciplinary Research in Mechanic, 3:2544-2545, 2016.

21. S. Mori. Introduction to Diffusion Tensor Imaging. Elsevier, Amsterdam, 2007.

22. M. E. Moseley, J. Kucharczyk, J. Mintorovitch, Y. Cohen, J. Kurhanewicz, N. Derugin, H. Asgari, and D. Norman. Diffusion-weighted MR imaging of acute stroke: correlation with T2-weighted and magnetic susceptibility-enhanced MR imaging in cats. American Journal of Neuroradiology, 11(3):423429, 1990.

23. S. Moskow and M. Vogelius. First-order corrections to the homogenized eigenvalues of a periodic composite medium. aconvergence proof. Proc. Roy. Soc. Edin., 127(6):1263-1278, 1997.

24. D. S. Novikov and V. G. Kiselev. Effective medium theory of a diffusion-weighted signal. NMR in Biomedicine, 23(7):682-697, 2010.

25. E. Ozarslan and T. H. Mareci. Generalized diffusion tensor imaging and analytical relationships between diffusion tensor imaging and high angular resolution diffusion imaging. Magnetic Resonance in Medicine, 50(5):955-65, 2003.

26. William S. Price. Pulsed-field gradient nuclear magnetic resonance as a tool for studying translational diffusion: Part 1. basic theory. Concepts Magn. Reson., 9(5):299-336, 1997.

27. D. Rohmer, A. Sitek, and G. T. Gullberg. Reconstruction and visualization of fiber and sheet structure with regularized tensor diffusion MRI in the human heart. Lawrence Berkeley National Laboratory Publication. LBNL-60277, 2006. 
28. F. Santosa and W. Synes. A dispersive effective medium for wave propagation in periodic composites. SIAM J. Appl. Math, 51:984-1005, 1991.

29. H. C. Torrey. Bloch equations with diffusion terms. Physical Review Online Archive (Prola), 104(3):563-565, 1956.

30. J. Veraart, D. S. Novikov, and E. Fieremans. TE dependent diffusion imaging (TEdDI) distinguishes between compartmental T2 relaxation times. NeuroImage, 182:360-369, 2018.

31. J. Veraart, D. H. Poot, W. Van Hecke, I. Blockx, A. Van der Linden, M. Verhoye, and J. Sijbers. More accurate estimation of diffusion tensor parameters using diffusion kurtosis imaging. Magnetic Reasonance in Medicine, 65(1):138-145, 2011.

32. S. Warach, D. Chien, W. Li, M. Ronthal, and R. R. Edelman. Fast magnetic resonance diffusionweighted imaging of acute human stroke. Neurology, 42(9):1717-1723, 1992. 